\title{
Computing knot Floer homology in cyclic branched covers
}

\author{
AdAM Simon LEVINE
}

\begin{abstract}
We use grid diagrams to give a combinatorial algorithm for computing the knot Floer homology of the pullback of a knot $K \subset S^{3}$ in its $m$-fold cyclic branched cover $\Sigma_{m}(K)$, and we give computations when $m=2$ for over fifty three-bridge knots with up to eleven crossings.
\end{abstract}

57R58; 57M12, 57M27

\section{Introduction}

Heegaard Floer knot homology, developed by Ozsváth and Szabó [15] and independently by Rasmussen [18], associates to a nulhomologous knot $K$ in a three-manifold $Y$ a group $\widehat{\mathrm{HFK}}(Y, K)$ that is an invariant of the knot type of $K$. If $K$ is a knot in $S^{3}$, then the inverse image of $K$ in $\Sigma_{m}(K)$, the $m$-fold cyclic branched cover of $S^{3}$ branched along $K$, is a nulhomologous knot $\widetilde{K}$ whose knot type depends only on the knot type of $K$, so the group $\widehat{\mathrm{HFK}}\left(\Sigma_{m}(K), \tilde{K}\right)$ is a knot invariant of $K$. In this paper, we describe an algorithm that can compute $\widehat{\operatorname{HFK}}\left(\Sigma_{m}(K), \widetilde{K}\right)$ (with coefficients in $\mathbb{Z} / 2$ ) for any knot $K \subset S^{3}$, and we give computations for a large collection of knots with up to eleven crossings.

Any knot $K \subset S^{3}$ can be represented by means of a grid diagram, consisting of an $n \times n$ grid in which the centers of certain squares are marked $X$ or $O$, such that each row and each column contains exactly one $X$ and one $O$. To recover a knot projection, draw an arc from the $X$ to the $O$ in each column and from the $O$ to the $X$ in each row, making the vertical strand pass over the horizontal strand at each crossing. We may view the diagram as lying on a standardly embedded torus $T^{2} \subset S^{3}$ by making the standard edge identifications; the horizontal grid lines become $\alpha$ circles and the vertical ones $\beta$ circles. Manolescu, Ozsváth, and Sarkar [12] showed that such diagrams can be used to compute $\widehat{\mathrm{HFK}}\left(S^{3}, K\right)$ combinatorially; we shall use them to give a combinatorial description of the chain complex for $\widehat{\operatorname{HFK}}\left(\Sigma_{m}(K), \widetilde{K}\right)$ for any knot $K \subset S^{3}$.

Let $m \geq 2$ and let $\tilde{T}$ be the surface obtained by gluing together $m$ copies of $T$ (denoted $T_{0}, \ldots, T_{m-1}$ ) along branch cuts connecting the $X$ and the $O$ in each 

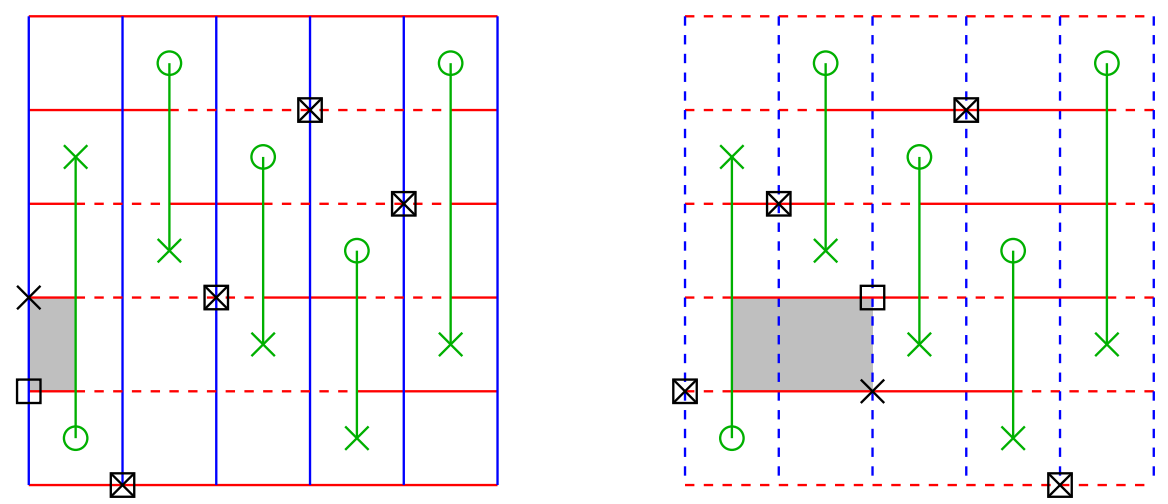

Figure 1: Heegaard diagram $\widetilde{D}=(\widetilde{T}, \widetilde{\boldsymbol{\alpha}}, \widetilde{\boldsymbol{\beta}}, \widetilde{\mathbf{w}}, \widetilde{\mathbf{z}})$ for $\left(\Sigma_{2}(K), \widetilde{K}\right)$, where $K$ is the right-handed trefoil. The solid and dashed lines represent different lifts of the $\alpha$ (horizontal/red) and $\beta$ (vertical/blue) circles. The black squares and crosses represent two generators of $\widetilde{C}=\mathrm{C} \widehat{\mathrm{FK}}(\widetilde{\mathcal{D}})$, and the shaded region is a disk that contributes to the differential.

column. Specifically, in each column, if the $X$ is above the $O$, then glue the left side of the branch cut in $T_{k}$ to the right side of the same cut in $T_{k+1}$ (indices modulo $m$ ); if the $O$ is above the $X$, then glue the left side of the branch cut in $T_{k}$ to the right side of the same cut in $T_{k-1}$. The obvious projection $\pi: \widetilde{T} \rightarrow T$ is an $m$-fold cyclic branched cover, branched around the marked points. Each $\alpha$ and $\beta$ circle in $T$ intersects the branch cuts a total of zero times algebraically and therefore has $m$ distinct lifts to $T$, and each lift of each $\alpha$ circle intersects exactly one lift of each $\beta$ circle. (We will describe these intersections more explicitly in Section 4.)

Denote by $\mathcal{R}$ the set of embedded rectangles in $T$ whose lower and upper edges are arcs of $\alpha$ circles, whose left and right edges are arcs of $\beta$ circles, and which do not contain any marked points in their interior. Each rectangle in $\mathcal{R}$ has $m$ distinct lifts to $\widetilde{T}$ (possibly passing through the branch cuts as in Figure 1); denote the set of such lifts by $\widetilde{\mathcal{R}}$.

Let $\mathcal{S}$ be the set of unordered $m n$-tuples $\mathbf{x}$ of intersection points between the lifts of $\alpha$ and $\beta$ circles such that each such lift contains exactly one point of $\mathbf{x}$. (We will give a more explicit characterization of the elements of $\mathcal{S}$ later.) Let $C$ be the $\mathbb{Z} / 2$-vector space generated by $\mathcal{S}$. Define a differential $\partial$ on $C$ by making the coefficient of $\mathbf{y}$ in $\widetilde{\partial} \mathbf{x}$ nonzero if and only if the following conditions hold.

- All but two of the points in $\mathbf{x}$ are also in $\mathbf{y}$. 
- There is a rectangle $R \in \widetilde{\mathcal{R}}$ whose lower-left and upper-right corners are in $\mathbf{x}$, whose upper-left and lower-right corners are in $\mathbf{y}$, and which does not contain any $X, O$, or point of $\mathbf{x}$ in its interior.

In Section 4, we shall define two gradings (Alexander and Maslov) on $C$, as well as a decomposition of $C$ as a direct sum of complexes corresponding to $\operatorname{spin}^{c}$ structures on $\Sigma_{m}(K)$. We shall prove the following theorem.

Theorem 1.1 The homology of the complex $(C, \partial)$ is isomorphic as a bigraded group to $\widehat{\operatorname{HFK}}\left(\Sigma_{m}(K), \widetilde{K} ; \mathbb{Z} / 2\right) \otimes V^{\otimes n-1}$, where $V \cong \mathbb{Z} / 2 \oplus \mathbb{Z} / 2$ with generators in bigradings $(0,0)$ and $(-1,-1)$.

In Section 2, we review the construction of knot Floer homology using multi-pointed Heegaard diagrams. In Section 3, we show how to obtain a Heegaard diagram for $\left(\Sigma_{m}(K), \widetilde{K}\right)$ given one for $\left(S^{3}, K\right)$, and we apply that discussion to grid diagrams in Section 4, proving Theorem 1.1. In Section 5, we give the values of $\widehat{\operatorname{HFK}}\left(\Sigma_{m}(K), \widetilde{K}\right)$ for over fifty knots with up to eleven crossings. (Grigsby [6] has shown how to compute these groups for two-bridge knots, so our tables only include knots that are not twobridge.) Finally, we make some observations and conjectures about these results in Section 6.

Acknowledgments I am grateful to Peter Ozsváth for suggesting this problem, providing lots of guidance, and reading a draft of this paper, and to John Baldwin, Josh Greene, Matthew Hedden, Robert Lipshitz, Tom Peters, and especially Eli Grigsby for many extremely helpful conversations. I would also like to thank the referees for their suggestions.

\section{Review of knot Floer homology}

Let us briefly recall the basic construction of knot Floer homology using multiple basepoints (Ozsváth-Szabó [15], Manolescu-Ozsváth-Sarkar [12] and Sarkar-Wang [20]). For simplicity, we work with coefficients modulo 2. A multi-pointed Heegaard diagram $\mathcal{D}=(\Sigma, \boldsymbol{\alpha}, \boldsymbol{\beta}, \mathbf{w}, \mathbf{z})$ consists of an oriented surface $\Sigma ;$ two sets of closed, embedded, piecewise disjoint curves $\boldsymbol{\alpha}=\left\{\alpha_{1}, \ldots, \alpha_{g+n-1}\right\}$ and $\boldsymbol{\beta}=\left\{\beta_{1}, \ldots, \beta_{g+n-1}\right\}$ (where $g=g(\Sigma)$ and $n \geq 1$ ), each of which spans a $g$-dimensional subspace of $H_{1}(\Sigma ; \mathbb{Z})$; and two sets of basepoints, $\mathbf{w}=\left\{w_{1}, \ldots, w_{n}\right\}$ and $\mathbf{z}=\left\{z_{1}, \ldots, z_{n}\right\}$, such that each component of $\Sigma-\bigcup \alpha_{i}$ and each component of $\Sigma-\bigcup \beta_{i}$ contains exactly one point of $\mathbf{w}$ and one point of $\mathbf{z}$. We call the components of $\Sigma-\bigcup \alpha_{i}-\bigcup \beta_{i}$ regions and denote them $R_{1}, \ldots, R_{N}$. The $\alpha$ and $\beta$ curves specify a Heegaard decomposition 
$H_{\alpha} \cup_{\Sigma} H_{\beta}$ for a 3-manifold $Y$, oriented so that $\Sigma$ acquires its orientation as $\partial H_{\alpha}$. We obtain a knot or link $K$ by connecting the $w$ (resp. $z$ ) basepoints to the $z$ (resp. $w$ ) basepoints with arcs in the complement of the $\alpha$ (resp. $\beta$ ) curves and push those arcs into $H_{\alpha}$ (resp. $H_{\beta}$ ). The orientations are such that $K$ intersects $\Sigma$ positively at the $z$ basepoints and negatively at the $w$ basepoints. In terms of Morse theory, the Heegaard diagram corresponds to a self-indexing Morse function $f$ on $Y$ with $n$ critical points of index $0, g+n-1$ of index $1, g+n-1$ of index 2 , and $n$ of index 3 . Given a Riemannian metric $g$, the knot $K$ is given as a union of gradient flowlines connecting the index 0 and 3 critical points through the $w$ and $z$ basepoints. We shall always assume that the knot $K$ is nulhomologous.

Let $\widehat{\mathrm{CFK}}(\mathcal{D})$ be the $\mathbb{Z} / 2$-vector space generated by the intersection points between the $(g+n-1)$-dimensional tori $\mathbb{T}_{\alpha}=\alpha_{1} \times \cdots \times \alpha_{g+n-1}$ and $\mathbb{T}_{\beta}=\beta_{1} \times \cdots \times \beta_{g+n-1}$ in the symmetric product $\operatorname{Sym}^{g+n-1}(\Sigma)$. The differential $\partial$ is defined by taking counts of holomorphic disks connecting intersection points:

$$
\partial \mathbf{x}=\sum_{\mathbf{y} \in \mathbb{T}_{\alpha} \cap \mathbb{T}_{\beta}} \sum_{\substack{\phi \in \pi_{2}(\mathbf{x}, \mathbf{y}) \mid \\ \mu(\phi)=1 \\ n_{\mathbf{w}}(\phi)=n_{\mathbf{z}}(\phi)=0}} \#(\widehat{\mathcal{M}}(\phi)) \mathbf{y} .
$$

Each homotopy class of Whitney disks $\phi \in \pi_{2}(\mathbf{x}, \mathbf{y})$ has an associated domain in $\Sigma$ : a 2-chain $D(\phi)=\sum a_{i} R_{i}$, such that $\partial D$ is made of arcs of $\alpha$ curves that connect each point of $\mathbf{x}$ to a point of $\mathbf{y}$ and arcs of $\beta$ curves that connect each point of $\mathbf{y}$ to a point of $\mathbf{x}$. Then $n_{\mathbf{w}}(\phi)$ (resp. $\left.n_{\mathbf{z}}(\phi)\right)$ is the sum of the multiplicities of the regions containing points of $\mathbf{w}$ (resp. $\mathbf{z}$ ). The Maslov index $\mu(\phi)$ can be given by formula due to Lipshitz [10]:

$$
\mu(\phi)=e(D)+p_{\mathbf{x}}(\mathcal{D})+p_{\mathbf{y}}(\mathcal{D}),
$$

where $e(D)$ is the Euler measure of $D$ and $p_{\mathbf{x}}(D)$ (resp. $\left.p_{\mathbf{y}}(D)\right)$ is the sum, taken over all points $x \in \mathbf{x}$ (resp. $y \in Y$ ), of the average of the multiplicities of the four domains that come together at $x$ (resp. $y$ ). The coefficient of $\mathbf{y}$ represents the number of holomorphic representatives of $\phi$ and generally depends on the choice of almost complex structure on $\Sigma$.

Each generator $\mathbf{x}$ has an associated $\operatorname{spin}^{c}$ structure $\mathfrak{s}_{\mathbf{w}}(\mathbf{x}) \in \operatorname{Spin}^{c}(Y)$, obtained by considering the gradient of a compatible Morse function outside of regular neighborhoods of flowlines through the points of $\mathbf{x}$ and $\mathbf{w}$. Given two generators $\mathbf{x}$ and $\mathbf{y}$, let $\gamma_{\mathbf{x}, \mathbf{y}}$ be any 1-cycle obtained by connecting $\mathbf{x}$ to $\mathbf{y}$ along the $\alpha$ circles and $\mathbf{y}$ to $\mathbf{x}$ along the $\beta$ circles, and let $\epsilon(\mathbf{x}, \mathbf{y})$ be its image in

$$
H_{1}(Y) \cong H_{1}(\Sigma) / \operatorname{Span}\left(\left[\alpha_{i}\right],\left[\beta_{i}\right] \mid i=1, \ldots, g+n-1\right) .
$$


Then $\mathfrak{s}_{w}(\mathbf{x})=\mathfrak{s}_{w}(\mathbf{y})$ if and only if $\epsilon(\mathbf{x}, \mathbf{y})=0$. The complex $\widehat{\mathrm{CFK}}(\mathcal{D})$ splits as a direct sum over $\mathfrak{s} \in \operatorname{Spin}^{c}(Y)$ of subcomplexes $\widehat{\mathrm{CFK}}(\mathcal{D}, \mathfrak{s})$, each generated by those $\mathbf{x} \in \mathbb{T}_{\alpha} \cap \mathbb{T}_{\beta}$ with $\mathfrak{s}_{w}(\mathbf{x})=\mathfrak{s}$.

The restriction of $\mathfrak{s}_{\mathbf{w}}(\mathbf{x})$ to $Y-K$ extends uniquely to a $\operatorname{spin}^{c}$ structure $\underline{\mathfrak{s}}_{\mathbf{w}, \mathbf{z}}(\mathbf{x})$ on the zero-surgery $Y_{0}(K)$. Given a Seifert surface $F$ for $K$, we define the Alexander grading of $\mathbf{x}$ (relative to $F$ ) as $A(\mathbf{x})=\frac{1}{2}\left\langle c_{1}\left(\underline{\mathfrak{s}}_{\mathbf{w}, \mathbf{z}}(\mathbf{x})\right),[\widehat{F}]\right)$, where $\widehat{F}$ is an extension of $F$ to $Y_{0}(K)$. This quantity is in independent of the choice of $F$ up to an additive constant, and it is completely well-defined if $Y$ is a rational homology sphere. The relative Alexander grading between two generators $\mathbf{x}$ and $\mathbf{y}, A(\mathbf{x}, \mathbf{y})=A(\mathbf{x})-A(\mathbf{y})$, can also be given as the linking number of $\gamma_{\mathbf{x}, \mathbf{y}}$ and $K$ (ie the intersection number of $\gamma_{\mathbf{x}, \mathbf{y}}$ with $\left.F\right)$, or by the formula $A(\mathbf{x}, \mathbf{y})=n_{\mathbf{z}}(D)-n_{\mathbf{w}}(D)$ when $\mathbf{x}$ and $\mathbf{y}$ are in the same $\operatorname{spin}^{c}$ structure and $\mathcal{D}$ is any domain connecting $\mathbf{x}$ to $\mathbf{y}$. The latter formula shows that the complex $\widehat{\mathrm{CFK}}(\mathcal{D})$ splits according to Alexander gradings.

When $Y$ is a rational homology sphere, the complex $\widehat{\mathrm{CFK}}(\mathcal{D})$ admits an absolute $\mathbb{Q}$-grading, the Maslov grading, which restricts to a relative $\mathbb{Z}$-grading on each $\widehat{\mathrm{CFK}}(\mathcal{D}, \mathfrak{s}){ }^{1}$ The relative Maslov grading between two generators $\mathbf{x}$ and $\mathbf{y}$ with $\mathfrak{s}_{\mathbf{w}}(\mathbf{x})=\mathfrak{s}_{\mathbf{w}}(\mathbf{y})$ is given by the integer $M(\mathbf{x}, \mathbf{y})=\mu(D)-2 n_{\mathbf{w}}(D)$, where $D$ is any domain connecting $\mathbf{x}$ to $\mathbf{y}$. The differential lowers this grading by 1 , so the grading descends to $\widehat{\mathrm{HFK}}(Y, K)$. The relative $\mathbb{Q}$-grading between generators in different $\operatorname{spin}^{c}$ structures can be computed using a formula of Lipshitz and Lee [9].

Theorem 2.1 ([15; 12;20]) For a suitable choice of complex structure, the homology of the complex $(\widehat{\mathrm{CFK}}(\mathcal{D}), \partial)$ is isomorphic to $\widehat{\mathrm{HFK}}(Y, K) \otimes V^{\otimes n-1}$, where $V \cong$ $\mathbb{Z} / 2 \oplus \mathbb{Z} / 2$ with generators in bigradings $(-1,-1)$ and $(0,0)$, and $\widehat{\operatorname{HFK}}(Y, K)$ is an invariant of the knot type of $K \subset Y$.

Call a diagram $\mathcal{D}$ nice if every elementary domain that does not contain a basepoint is either a bigon or a square. According to results of Manolescu-Ozsváth-Sarkar [12] and Sarkar-Wang [20], the holomorphic disks are easy to describe when $\mathcal{D}$ is nice.

Theorem 2.2 Let $\mathcal{D}$ be a nice diagram, and let $\phi \in \pi_{2}(\mathbf{x}, \mathbf{y})$ be a Whitney disk in $\mathcal{D}$ with $\mu(\phi)=1$. Then $\phi$ admits a holomorphic representative if and only if $D(\phi)$ is either a bigon or a rectangle without any basepoint or point of $\mathbf{x}$ in its interior.

It follows that when $\mathcal{D}$ is nice, the coefficients $\# \widehat{\mathcal{M}}(\phi)$ in the boundary map can be determined from the combinatorics of the diagram, without reference to the choice of complex structure on $\Sigma$, so $\widehat{\operatorname{HFK}}(Y, K)$ can be computed algorithmically.

\footnotetext{
${ }^{1}$ More generally, such a grading can be defined on $\widehat{\mathrm{CFK}}(\mathcal{D}, \mathfrak{s})$ whenever $c_{1}(\mathfrak{s})$ is torsion.
} 
If $K$ is a knot in $S^{3}$, then a grid diagram for $K$, drawn on a torus as in Section 1, yields a Heegaard diagram $\mathcal{D}=\left(T^{2}, \boldsymbol{\alpha}, \boldsymbol{\beta}, \mathbf{w}, \mathbf{z}\right)$ for the pair $\left(S^{3}, K\right)$, where the $\alpha$ circles are the horizontal lines of the grid, the $\beta$ circles are the vertical lines, and the $w$ and $z$ basepoints are the points marked $O$ and $X$, respectively. Every region of this diagram is a rectangle, so $\widehat{\mathrm{HFK}}\left(S^{3}, K\right)$ can be computed combinatorially as above. Specifically, the generators correspond to permutations of the set $\{1, \ldots, n\}$, and the Alexander and Maslov gradings of each generator can be given by simple formulae (discussed later). Using this diagram, Baldwin and Gillam [1] have computed $\widehat{\mathrm{HFK}}\left(S^{3}, K\right)$ for all knots with up to 12 crossings. Additionally, Manolescu, Ozsváth, Szabó, and Thurston [13] give a self-contained proof that this construction yields a knot invariant. (See also Sarkar and Wang [20], who show how to obtain good diagrams for knots in arbitrary 3-manifolds.)

\section{Heegaard diagrams for cyclic branched covers of knots}

Given a knot $K \subset S^{3}$ and an integer $m \geq 2$, the cover of $S^{3}-K$ corresponding to the canonical homeomorphism $\pi_{1}\left(S^{3}-K\right) \rightarrow \mathbb{Z} / m$ extends to an $m$-sheeted branched cover $\pi: \Sigma_{m}(K) \rightarrow S^{3}$, the $m$-fold cyclic branched cover, whose downstairs branch locus is $K$ and whose upstairs branch locus is a knot $\widetilde{K} \subset \Sigma_{m}(K)$. The manifold $\Sigma_{m}(K)$ can be constructed explicitly from $m$ copies of $S^{3}-$ int $F$, where $F$ is a Seifert surface for $K$, by connecting the negative side of a bicollar of $F$ in the $k$ th copy to the positive side in the $(k+1)$ th (indices modulo $m$ ). The inverse image of $K$ in $\Sigma_{m}(K)$ is a knot $\widetilde{K}$, which is nulhomologous because it bounds a Seifert surface (any of the lifts of the original Seifert surface $F$ ). This construction does not depend on the choice of Seifert surface. For details, see Rolfsen [19, chapters 6, 10].

The group of covering transformations of $\Sigma_{m}(K) \rightarrow S^{3}$ is cyclic of order $m$, generated by a map $\tau_{m}: \Sigma_{m}(K) \rightarrow \Sigma_{m}(K)$ that takes the $k$ th copy of $S^{3}-$ int $F$ to the $(k+1)$ th (indices modulo $m$ ). If $\gamma$ is a 1 -cycle in $S^{3}$, then by using transfer homomorphisms, we see that for any lift $\tilde{\gamma}$, the equation

$$
\sum_{k=0}^{m-1} \tau_{m *}^{k}(\tilde{\gamma})=0
$$

holds in $H_{1}\left(\Sigma_{m}(K) ; \mathbb{Z}\right)$. In particular, when $m=2$, we have $\tau_{2 *}(\tilde{\gamma})=-\tilde{\gamma}$.

When $m$ is a power of a prime $p$, the group $H_{1}\left(\Sigma_{m}(K) ; \mathbb{Z}\right)$ is then finite and contains no $p^{r}$-torsion for any $r$ (Gordon [5, page 16]). The order of $H_{1}\left(\Sigma_{m}(K)\right)$ is equal to $\prod_{j=0}^{m-1} \Delta_{K}\left(\omega^{j}\right)$, where $\Delta_{K}$ is the Alexander polynomial of $K$, and $\omega$ is a primitive $m$ th root of unity (Fox [4, page 149]). In particular, note that the action of the deck 
transformation group on $H_{1}\left(\Sigma_{m}(K) ; \mathbb{Z}\right)$ has no nonzero fixed points: if $\tau_{m *}(\alpha)=\alpha$, then

$$
0=\alpha+\tau_{m *}(\alpha)+\cdots+\tau_{m *}^{m-1}(\alpha)=m \alpha,
$$

by (1), so $\alpha=0$.

Let $\mathcal{D}=(S, \boldsymbol{\alpha}, \boldsymbol{\beta}, \mathbf{w}, \mathbf{z})$ be a multi-pointed Heegaard diagram for $K \subset S^{3}$ with genus $g$ and $n$ basepoint pairs. ${ }^{2}$ If $f: S^{3} \rightarrow \mathbb{R}$ is a self-indexing Morse function compatible with $\mathcal{D}$, then $\tilde{f}=f \circ \pi: \Sigma_{m}(K) \rightarrow \mathbb{R}$ is a self-indexing Morse function for the pair $\left(\Sigma_{m}(K), \widetilde{K}\right)$ whose critical points are simply the inverse images of the critical points of $f$. This function induces a Heegaard splitting $\Sigma_{m}(K)=\widetilde{H}_{\alpha} \cup_{\widetilde{S}} \widetilde{H}_{\beta}$ that projects onto the Heegaard splitting of $S^{3}$. A simple Euler characteristic argument shows that the genus of the new Heegaard surface $\widetilde{S}=\pi^{-1}(S)$ is $h=m g+(m-1)(n-1)$. Each $\alpha$ and $\beta$ circle in $S$ bounds a disk in $S^{3}-K$ and hence has $m$ distinct preimages in $\Sigma_{m}(K)$. Thus, we obtain a Heegaard diagram $\widetilde{\mathcal{D}}=(\widetilde{S}, \widetilde{\boldsymbol{\alpha}}, \widetilde{\boldsymbol{\beta}}, \widetilde{\mathbf{w}}, \widetilde{\mathbf{z}})$, where $\widetilde{S}$ is a surface of genus $h$ and $\widetilde{\boldsymbol{\alpha}}, \widetilde{\boldsymbol{\beta}}, \widetilde{\mathbf{w}}$, and $\widetilde{\mathbf{z}}$ are the inverse images of the corresponding objects under the covering map.

The generators of the complex $\widehat{\mathrm{CFK}}(\widetilde{\mathcal{D}})$ may be described as follows.

Lemma 3.1 Any generator $\mathbf{x}$ of $\widehat{\mathrm{CFK}}(\widetilde{\mathcal{D}})$ can be decomposed (non-uniquely) as $\mathbf{x}=\widetilde{\mathbf{x}}_{1} \cup \cdots \cup \widetilde{\mathbf{x}}_{m}$, where $\mathbf{x}_{1}, \ldots, \mathbf{x}_{m}$ are generators of $\widehat{\mathrm{CFK}}(\mathcal{D})$, and $\widetilde{\mathbf{x}}_{i}$ is a lift of $\mathbf{x}_{i}$ to $\tilde{\mathcal{D}}$.

Proof Given a generator $\mathbf{x}$ of $\widehat{\mathrm{CFK}}(\widetilde{\mathcal{D}})$, let $G_{\mathbf{x}}$ be a graph with vertices denoted $\left\{a_{1}, \ldots, a_{g+n-1}, b_{1}, \ldots, b_{g+n-1}\right\}$ and edges $\left\{e_{x} \mid x \in \mathbf{x}\right\}$, where $e_{x}$ connects $a_{i}$ to $b_{j}$ if $x$ is an intersection point between lifts of $\alpha_{i}$ and $\beta_{j}$. This is clearly a bipartite graph in which each vertex has incidence number $m$. By König's Theorem [3, Proposition 5.3.1], the edges of $G_{\mathbf{x}}$ can be partitioned (non-uniquely) into $m$ perfect pairings, each of which corresponds to a lift of a generator of $\widehat{\mathrm{CFK}}(\mathcal{D})$.

Example 3.2 As will be explained in Section 4, the diagram $\widetilde{D}$ in Figure 1 is the double branched cover of a grid diagram $\mathcal{D}$ for the right-handed trefoil in $S^{3}$. The generator $\mathbf{x}$ of $\widehat{\mathrm{CFK}}(\widetilde{D})$ indicated by the crosses can be decomposed either as lifts of the generators $\mathbf{x}_{1}=(20143)$ and $\mathbf{x}_{2}=(13240)$ or as lifts of $\mathbf{x}_{1}^{\prime}=(23140)$ and $\mathbf{x}_{2}^{\prime}=(10243)$ (where we identify generators of $\mathcal{D}$ with permutations of $\{0,1,2,3,4\}$ as described by Manolescu, Ozsváth, and Sarkar [12]). This provides an example of the non-uniqueness of decompositions beyond reordering of the $\mathbf{x}_{i}$.

\footnotetext{
${ }^{2}$ We denote the Heegaard surface by $S$ rather than $\Sigma$ to avoid confusion with the notation $\Sigma_{m}(K)$.
} 
Given a generator $\mathbf{x}_{0}$ of $\widehat{\mathrm{CFK}}(\mathcal{D})$, let $L\left(\mathbf{x}_{0}\right)$ denote the generator of $\widehat{\mathrm{CFK}}(\widetilde{\mathcal{D}})$ consisting of all $m$ lifts of each point of $\mathbf{x}_{0}$. Using the action of the deck transformation $\tau_{m}$ on $\mathcal{D}$, we may write $L\left(\mathbf{x}_{0}\right)=\widetilde{\mathbf{x}}_{0} \cup \tau_{m}\left(\widetilde{\mathbf{x}}_{0}\right) \cup \cdots \cup \tau_{m}^{m-1}\left(\widetilde{\mathbf{x}}_{0}\right)$, where $\widetilde{\mathbf{x}}_{0}$ is any lift of $\mathbf{x}_{0}$ to $\widetilde{D}$

Lemma 3.3 All generators of $\widehat{\mathrm{CFK}}(\widetilde{\mathcal{D}})$ of the form $\mathbf{x}=L\left(\mathbf{x}_{0}\right)$ are in the same $\operatorname{spin}^{c}$ structure, denoted $\mathfrak{s}_{0}$ and called the canonical $\operatorname{spin}^{c}$ structure on $\Sigma_{m}(K)$.

Proof (Adapted from Grigsby [7].) Let $\mathbf{x}_{0}$ and $\mathbf{y}_{0}$ be generators of $\widehat{\mathrm{CFK}}(\mathcal{D})$; we shall show that $L\left(\mathbf{x}_{0}\right)$ and $L\left(\mathbf{y}_{0}\right)$ are in the same $\operatorname{spin}^{c}$ structure. Let $\gamma_{\mathbf{x}_{0}, \mathbf{y}_{0}}$ be a 1-cycle joining $\mathbf{x}_{0}$ and $\mathbf{y}_{0}$ as in Section 2, and let $\tilde{\gamma}_{\mathbf{x}_{0}, \mathbf{y}_{0}}$ be a lift of $\gamma_{\mathbf{x}_{0}, \mathbf{y}_{0}}$ to $\widetilde{S}$. Then the 1-cycle

$$
\tilde{\gamma}_{\mathbf{x}_{0}, \mathbf{y}_{0}}+\tau_{m *}\left(\tilde{\gamma}_{\mathbf{x}_{0}, \mathbf{y}_{0}}\right)+\cdots+\tau_{m *}^{m-1}\left(\tilde{\gamma}_{\mathbf{x}_{0}, \mathbf{y}_{0}}\right)
$$

connects $L\left(\mathbf{x}_{0}\right)$ and $L\left(\mathbf{y}_{0}\right)$. Then $\epsilon\left(L\left(\mathbf{x}_{0}\right), L\left(\mathbf{y}_{0}\right)\right)=0$ by (1), so $L\left(\mathbf{x}_{0}\right)$ and $L\left(\mathbf{y}_{0}\right)$ are in the same $\operatorname{spin}^{c}$ structure.

Remark 3.4 Note that the $\operatorname{spin}^{c}$ structure $\mathfrak{s}_{0}$ is fixed under the action of $\tau_{m}$. To see this, if $f: S^{3} \rightarrow \mathbb{R}$ is a self-indexing Morse function for $\left(S^{3}, K\right)$, its pullback $\tilde{f}: \Sigma_{m}(K) \rightarrow \mathbb{R}$ is $\tau_{m}$-invariant. Using a Riemannian metric on $\Sigma_{m}(K)$ that is the pullback of a metric on $S^{3}$, the gradient $\vec{\nabla} \tilde{f}$ is $\tau_{m}$-invariant and projects onto $\vec{\nabla} f$, and the flowlines for $\tilde{f}$ are precisely the lifts of flowlines for $f$. If $N$ is the union of neighborhoods of flowlines through the points of $\mathbf{x}_{0}$ and $\mathbf{w}$, where $\mathbf{x}_{0}$ is a generator of $\widehat{\mathrm{CFK}}(\mathcal{D})$, then $\tilde{N}=\pi^{-1}(N)$ is the union of neighborhoods of flowlines through the points of $L\left(\mathbf{x}_{0}\right)$ and $\tilde{\mathbf{w}}$. By suitably modifying $\vec{\nabla} \tilde{f}$ on $\tilde{N}$, we may obtain a $\tau_{m}$-invariant vector field that determines $\mathfrak{s}_{\tilde{\mathbf{w}}}\left(L\left(\mathbf{x}_{0}\right)\right)=\mathfrak{s}_{0} \cdot{ }^{3}$ Now, if $m$ is a prime power, then this property uniquely characterizes $\mathfrak{s}_{0}$, for if $\mathfrak{s}_{0}^{\prime}$ is another $\operatorname{spin}^{c}$ structure fixed under the action of $\tau_{m}$, then the difference between $\mathfrak{s}_{0}$ and $\mathfrak{s}_{0}^{\prime}$ is a class in $H_{1}\left(\Sigma_{m}(K) ; \mathbb{Z}\right)$ that is fixed by $\tau_{m}$ and hence equals zero. For more about the significance of $\mathfrak{s}_{0}$, see Grigsby, Ruberman, and Strle [8].

Proposition 3.5 If $\mathbf{x}=\widetilde{\mathbf{x}}_{1} \cup \cdots \cup \widetilde{\mathbf{x}}_{m}$ as in Lemma 3.1, then the Alexander grading of $\mathbf{x}$ (computed with respect to a lift of a Seifert surface for $K$ ) is equal to the average of the Alexander gradings of $\mathbf{x}_{1}, \ldots, \mathbf{x}_{m} \cdot{ }^{4}$ In particular, for any generator $\mathbf{x}_{0}$ of $\widehat{\mathrm{CFK}}(\mathcal{D})$, we have $A\left(\mathbf{x}_{0}\right)=A\left(L\left(\mathbf{x}_{0}\right)\right)$.

\footnotetext{
${ }^{3}$ In general, spin ${ }^{c}$ structures can always be pulled back under a local diffeomorphism using the vector field interpretation. Specifically, if $F: M \rightarrow N$ is a local diffeomorphism and $\xi$ is a nonvanishing vector field on $N$ that determines a given $\operatorname{spin}^{c}$ structure $\mathfrak{s} \in \operatorname{Spin}^{c}(N)$, then $F^{*}(\mathfrak{s}) \in \operatorname{Spin}^{c}(M)$ is determined by the vector field $\left(F_{*}\right)^{-1}(\xi)$. The first Chern class is natural under this pullback.

${ }^{4}$ Note that we have specified a Seifert surface in order to define the Alexander grading. When $m$ is a prime power, however, $\Sigma_{m}(K)$ is a rational homology sphere, so the Alexander grading does not depend at all on the choice of Seifert surface.
} 
Proof We first consider the relative Alexander gradings. Let $F \subset S^{3}$ be a Seifert surface for $K$, and let $\widetilde{F}$ be a lift of $F$ to $\Sigma_{m}(K)$. The translates $\widetilde{F}, \tau_{m}(\widetilde{F}), \ldots, \tau_{m}^{m-1}(\widetilde{F})$ are all Seifert surfaces for $\widetilde{K}$. The relative Alexander grading between two generators does not depend on the choice of Seifert surface, so for generators $\mathbf{x}, \mathbf{y}$ of $\widehat{\mathrm{CFK}}(\widetilde{D})$, we have

$$
m A(\mathbf{x}, \mathbf{y})=\gamma_{\mathbf{x}, \mathbf{y}} \cdot \tilde{F}+\gamma_{\mathbf{x}, \mathbf{y}} \cdot \tau_{m}(\tilde{F})+\cdots+\gamma_{\mathbf{x}, \mathbf{y}} \cdot \tau_{m}^{m-1}(\tilde{F}),
$$

where $\gamma_{\mathbf{x}, \mathbf{y}}$ is a 1-cycle connecting $\mathbf{x}$ and $\mathbf{y}$ as above. The projection $\pi_{*}\left(\gamma_{\mathbf{x}, \mathbf{y}}\right)$ is a 1-cycle in $S$ that goes from points of $\pi(\mathbf{x})$ to points of $\pi(\mathbf{y})$ along $\alpha$ circles and from points of $\pi(\mathbf{y})$ to points of $\pi(\overline{\mathbf{x}})$ along $\beta$ circles. Every intersection point of $\gamma_{\mathbf{x}, \mathbf{y}}$ with one of the lifts of $F$ corresponds to an intersection point of $\pi_{*}\left(\gamma_{\mathbf{x}, \mathbf{y}}\right)$ with $F$, so

$$
\gamma_{\mathbf{x}, \mathbf{y}} \cdot \tilde{F}+\gamma_{\mathbf{x}, \mathbf{y}} \cdot \tau_{m}(\tilde{F})+\cdots+\gamma_{\mathbf{x}, \mathbf{y}} \cdot \tau_{m}^{m-1}(\tilde{F})=\pi_{*}\left(\gamma_{\mathbf{x}, \mathbf{y}}\right) \cdot F
$$

The restriction of $\pi_{*}\left(\gamma_{\mathbf{x}, \mathbf{y}}\right)$ to any $\alpha$ or $\beta$ circle consists of $m$ (possibly constant or overlapping) arcs. By perhaps adding copies of the $\alpha$ or $\beta$ circle, we can arrange that these arcs connect a point of $\mathbf{x}_{1}$ with a point of $\mathbf{y}_{1}$, a point of $\mathbf{x}_{2}$ with a point of $\mathbf{y}_{2}$, and so on. In other words,

$$
\pi_{*}\left(\gamma_{\mathbf{x}, \mathbf{y}}\right) \equiv \gamma_{\mathbf{x}_{1}, \mathbf{y}_{1}}+\cdots+\gamma_{\mathbf{x}_{m}, \mathbf{y}_{m}}
$$

modulo the $\alpha$ and $\beta$ circles in $\mathcal{D}$, whose intersection numbers with $F$ are zero. We have:

$$
\begin{aligned}
A(\mathbf{x}, \mathbf{y}) & =\frac{1}{m}\left(\gamma_{\mathbf{x}_{1}, \mathbf{y}_{1}}+\ldots,+\gamma_{\mathbf{x}_{m}, \mathbf{y}_{m}}\right) \cdot F \\
& =\frac{1}{m}\left(A\left(\mathbf{x}_{1}, \mathbf{y}_{1}\right)+\cdots+A\left(\mathbf{x}_{m}, \mathbf{y}_{m}\right)\right) .
\end{aligned}
$$

Thus, the Alexander grading of a generator of $\widehat{\mathrm{CFK}}(\tilde{\mathcal{D}})$ is given up to an additive constant by the average Alexander grading of its parts.

To pin down the additive constant, note that the branched covering map $\pi: \Sigma_{m}(K) \rightarrow$ $S^{3}$ extends to an unbranched covering map from the zero-surgery on $\tilde{K}$ to the zerosurgery on $K, \pi_{0}: Y_{0}(\tilde{K}) \rightarrow S_{0}^{3}(K)$. Since this is a local diffeomorphism, it is possible to pull back $\operatorname{spin}^{c}$ structures. Let $\mathbf{x}_{0}$ be a generator of $\widehat{\mathrm{CFK}}(\mathcal{D})$ in Alexander grading 0 , and let $\mathbf{x}=L\left(\mathbf{x}_{0}\right)$. (The symmetry $\widehat{\operatorname{HFK}}\left(S^{3}, K, i\right) \cong \widehat{\operatorname{HFK}}\left(S^{3}, K,-i\right)$ and the fact that $\operatorname{rank} \widehat{\operatorname{HFK}}\left(S^{3}, K\right) \equiv \operatorname{det}(K) \equiv 1(\bmod 2)[15]$ imply that such $\widehat{\operatorname{HFK}}\left(S^{3}, K, 0\right)$ has odd rank, so such a generator $\mathbf{x}_{0}$ always exists.) As in the discussion following Lemma 3.3, we may find a nonvanishing vector field that determines $\mathfrak{s}_{\widetilde{\mathbf{w}}}(\mathbf{x})=\mathfrak{s}_{0}$ and is $\tau_{m}$-equivariant. The unique extension (up to isotopy) of this vector field to $\Sigma_{m}(K)_{0}$ can also be made $\tau_{m}$-invariant, so it is the pullback of an extension to $S_{0}^{3}$ of a vector field determining $\mathfrak{s}_{\mathbf{w}}\left(\mathbf{x}_{0}\right)$. It follows that $\left.\mathfrak{s}_{\tilde{\mathbf{w}}, \widetilde{\mathbf{z}}}(\mathbf{x})=\pi_{0}^{*} \underline{\mathfrak{s}}_{\mathbf{w}, \mathbf{z}}\left(\mathbf{x}_{0}\right)\right)$. Now, if $\widehat{\widetilde{F}} \subset Y_{0}(\widetilde{K})$ 
is obtained by capping off $\widetilde{F}$ in the zero-surgery, then $\pi_{0 *}[\widehat{\widetilde{F}}]=[\widehat{F}]$ in $H_{2}\left(S_{3}^{0} ; \mathbb{Z}\right)$. We therefore have:

$$
\begin{aligned}
A(\mathbf{x}) & =\frac{1}{2}\left\langle c_{1}\left(\underline{\mathfrak{s}}_{\tilde{\mathbf{w}}, \tilde{\mathbf{z}}}(\mathbf{x})\right),[\widehat{\widetilde{F}}]\right\rangle \\
& =\frac{1}{2}\left\langle c_{1}\left(\pi_{0}^{*}\left(\underline{\mathfrak{s}}_{\mathbf{w}, \mathbf{z}}\left(\mathbf{x}_{0}\right)\right)\right),[\widehat{\widetilde{F}}]\right\rangle \\
& =\frac{1}{2}\left\langle c_{1}\left(\underline{\mathfrak{s}}_{\mathbf{w}, \mathbf{z}}\left(\mathbf{x}_{0}\right)\right), \pi_{0 *}[\widehat{\widetilde{F}}]\right\rangle \\
& =\frac{1}{2}\left\langle c_{1}\left(\underline{\mathfrak{s}}_{\mathbf{w}, \mathbf{z}}\left(\mathbf{x}_{0}\right)\right),[\widehat{F}]\right\rangle \\
& =0=A\left(\mathbf{x}_{0}\right) .
\end{aligned}
$$

Thus, the additive constant $C$ must equal 0 .

Remark 3.6 When $K$ is a two-bridge knot and $m=2$, Grigsby [7] shows that for a specific diagram $\mathcal{D}$, the map $L$ is surjective and preserves the relative Maslov grading. Therefore, for any two-bridge knot $K, \widehat{\operatorname{HFK}}\left(\Sigma_{2}(K), \widetilde{K}, \mathfrak{s}_{0}\right) \cong \widehat{\operatorname{HFK}}\left(S^{3}, K\right)$, up to a possible shift in the absolute Maslov grading. It may be possible to extend this result to a wider class of knots, such as alternating knots. However, in general $L$ is neither surjective nor Maslov-grading-preserving.

Finally, we consider the regions in $\widetilde{D}$. First, note that the preimage of any region $R$ in $\mathcal{D}$ consists of either $m$ distinct regions, each of which is projected diffeomorphically onto $R$, or a single region. (In the former case, we say that $R$ is evenly covered.) In particular, when $\mathcal{D}$ is nice, each region of $\mathcal{D}$ that does not contain a basepoint is a simply-connected polygon that misses the branch set, so it is evenly covered. Thus, we obtain the following proposition.

Proposition 3.7 Let $\mathcal{D}$ be a nice Heegaard diagram for $\left(S^{3}, K\right)$, and let $\widetilde{D}$ be its $m$-fold cyclic branched cover. Then $\widetilde{\mathcal{D}}$ is nice.

\section{Grid diagrams and cyclic branched covers}

Proof of Theorem 1.1 As described in Section 1, any oriented knot $K \subset S^{3}$ can be represented by means of a grid diagram. By drawing the grid diagram on a standardly embedded torus in $S^{3}$, we may think of the grid diagram as a genus 1, multi-pointed Heegaard diagram $\mathcal{D}=\left(T^{2}, \boldsymbol{\alpha}, \boldsymbol{\beta}, \mathbf{w}, \mathbf{z}\right)$ for the pair $\left(S^{3}, K\right)$, where the $\alpha$ circles are the horizontal lines of the grid, the $\beta$ circles are the vertical lines, the $w$ basepoints are in the regions marked $O$, and the $z$ basepoints are in the regions marked $X$. 
Note that the diagram $\mathcal{D}$ is nice, so the differential can be computed combinatorially as described in Section 2. Specifically, the coefficient of $\mathbf{y}$ in $\partial \mathbf{x}$ is 1 if all but two of the points of $\mathbf{x}$ and $\mathbf{y}$ agree and there is a rectangle embedded in the torus with points of $\mathbf{x}$ as its lower-left and upper-right corners, points of $\mathbf{y}$ as its lower-right and upper-left corners, and no basepoints or points of $\mathbf{x}$ in its interior, and 0 otherwise. Note that there cannot be two such rectangles, or else $K$ would be a split link.

A Seifert surface for $K$ may be seen as follows. We may isotope $K$ to lie entirely within $H_{\alpha}$ by letting the arcs of $K \cap H_{\beta}$ fall onto the boundary torus. In fact, it lies within a ball contained in $H_{\alpha}$ since the knot projection in the grid diagram never passes through the left edge of the grid. Take a Seifert surface $F$ contained in this ball, and then isotope $F$ and $K$ so that $K$ returns to its original position. $F$ then intersects the Heegaard surface $T^{2}$ in $n$ arcs, one connecting the two basepoints in each column of the grid diagram, and it intersects $H_{\beta}$ in strips that lie above these arcs. The orientations of $K$ and $S^{3}$ imply that the positive side of a bicollar for $F$ lies on the right of one of these strips when the $X$ is above the $O$ and on the left when the $O$ is above the $X$.

If we construct $\Sigma_{m}(K)$ by gluing together $m$ copies of $S^{3}-$ int $F$ as in Section 3, the Heegaard surfaces in each copy are connected exactly to each other as described in Section 1 to form a surface $\widetilde{T}$. Hence, $\widetilde{\mathcal{D}}=(\widetilde{T}, \widetilde{\boldsymbol{\alpha}}, \widetilde{\boldsymbol{\beta}}, \widetilde{\mathbf{w}}, \widetilde{\mathbf{z}})$ is a Heegaard diagram for $\left(\Sigma_{m}(K), \widetilde{K}\right)$ for which the results of Section 3 apply. In particular, it is a nice Heegaard diagram.

It remains to show that the domains that count for the differential in $\widehat{\mathrm{CFK}}(\tilde{D})$ are precisely the lifts of those that count for the differential in $\widehat{\mathrm{CFK}}(\mathcal{D})$, as was asserted in Section 1. Since $\widetilde{D}$ is a nice diagram with no bigons, any domain that counts for the differential is an embedded rectangle $R$. The projection of $R$ to $\mathcal{D}, \pi(R)$, is an immersed rectangle in $\widetilde{D}$ whose edges are contained in at most two $\alpha$ circles and two $\beta$ circles. By lifting $\pi(R)$ to the universal cover of $T^{2}$, we see that $\pi(R)$ cannot intersect any $\alpha$ or $\beta$ circle more than once, or else it would contain an entire column or row of the grid diagram and hence a basepoint. Therefore, $\pi(R)$ is an embedded rectangle that misses the basepoints, so it counts for the differential of $\widehat{\mathrm{CFK}}(\mathcal{D})$.

We shall now give a more explicit description of the generators of $\widehat{\operatorname{CFK}}(\widetilde{D})$ and their gradings in order to facilitate computation.

In the grid diagram $\mathcal{D}$, we label the $\alpha$ circles $\alpha_{0}, \ldots, \alpha_{n-1}$ from bottom to top and the $\beta$ circles $\beta_{0}, \ldots, \beta_{n-1}$ from left to right. Each $\alpha$ circle intersects each $\beta$ circle exactly once: $\beta_{i} \cap \alpha_{j}=\left\{x_{i j}\right\}$. Generators of $\widehat{\mathrm{CFK}}(\mathcal{D})$ then correspond to permutations of the index set $\{0, \ldots, n-1\}$ via the correspondence $\sigma \mapsto\left(x_{0, \sigma(0)}, \ldots, x_{n-1, \sigma(n-1)}\right)$. 
For each grid point $x$, let $w(x)$ denote the winding number of the knot projection around $x$. Let $p_{1}, \ldots, p_{8 n}$ (repetitions allowed) denote the vertices of the $2 n$ squares containing basepoints, and set

$$
a=\frac{1-n}{2}+\frac{1}{8} \sum_{i=1}^{8 n} w\left(p_{i}\right) .
$$

According to Manolescu, Ozsváth, and Sarkar [12], the Alexander grading of a generator $\mathbf{x}$ of $\widehat{\mathrm{CFK}}(\mathcal{D})$ is given by the formula

$$
A(\mathbf{x})=a-\sum_{x \in \mathbf{x}} w(x) .
$$

There is also a formula for the Maslov grading of a generator, but it is not relevant for our purposes.

The generators of $\widehat{\mathrm{CFK}}(\widetilde{\mathcal{D}})$ can be described easily as follows. For any $i=0, \ldots, n-1$ and $j=0, \ldots, n-1$, each lift of $\beta_{i}$ meets exactly one lift of $\alpha_{j}$. Specifically, let $\widetilde{\beta}_{j}^{k}$ denote the lift of $\beta_{j}$ on the $k$ th copy of $\mathcal{D}$ (for $k=0, \ldots, m-1$ ). Let $\tilde{\alpha}_{j}^{k}$ denote the lift of $\alpha_{j}$ that intersects the leftmost edge of the $k$ th grid diagram $\left(\widetilde{\beta}_{0}^{k}\right)$. Let $\tilde{x}_{i, j}^{k}$ denote the lift of $x_{i, j}$ on the $k$ th diagram. Define a map $g: \mathbb{Z} / n \times \mathbb{Z} / n \times \mathbb{Z} / m \rightarrow \mathbb{Z} / m$ by $g(i, j, k)=k-w\left(x_{i, j}\right) \bmod m$. The lift of $\alpha_{j}$ that meets a particular $\widetilde{\beta}_{i}^{k}$ is given by the following lemma.

Lemma 4.1 The point $\tilde{x}_{i, j}^{k}$ is the intersection between $\widetilde{\beta}_{i}^{k}$ and $\widetilde{\alpha}_{j}^{g(i, j, k)}$.

Proof We induct on $i$. For $i=0$, we have $w\left(x_{0, j}\right)=0$, and by construction $\tilde{\alpha}_{j}^{k}$ meets $\widetilde{\beta}_{0}^{k}$. For the induction step, let $S$ be the segment of $\alpha_{j}$ from $x_{i, j}$ to $x_{i+1, j}$. Note that $w\left(x_{i+1, j}\right)$ is equal to $w\left(x_{i, j}\right)+1$ if $S$ passes below the $X$ and above the $O$ in its column, $w\left(x_{i, j}\right)-1$ if it passes above $X$ and below $O$, and $w\left(x_{i, j}\right)$ otherwise. Similarly, if $\tilde{x}_{i, j}^{k}$ lies on $\widetilde{\alpha}_{j}^{l}$, then by the previous discussion, $\tilde{x}_{i+1, j}^{k}$ lies on $\widetilde{\alpha}_{j}^{l-1}$ in the first case, on $\widetilde{\alpha}_{j}^{l+1}$ in the second, and on $\widetilde{\alpha}_{j}^{l}$ in the third (upper indices modulo $m$ ). This proves the induction step.

We may then identify the generators of $\widehat{\mathrm{CFK}}(\widetilde{\mathcal{D}})$ with the set of $m$-to-one maps

$$
\phi:\{0, \ldots, n-1\} \times\{0, \ldots, m-1\} \rightarrow\{0, \ldots, n-1\}
$$

such that for each $j=0, \ldots, n-1$, the function $g(\cdot, j, \cdot)$ assumes all $m$ possible values on $\phi^{-1}(j)$. In other words, if we shade the $m$ lifts of each $\alpha$ circle with different colors as in Figure 1 and arrange the copies of $\mathcal{D}$ horizontally, a generator is a 
selection of $m n$ grid points so each column contains one point and each row contains $m$ points, one of each color. It is not difficult to enumerate such maps algorithmically. To split up the generators of $\widehat{\mathrm{CFK}}(\widetilde{\mathcal{D}})$ according to $\operatorname{spin}^{c}$ structures, we simply need to express $\epsilon(\mathbf{x}, \mathbf{y})$ in terms of a $\mathbb{Z}$-module presentation for $H_{1}\left(\Sigma_{m}(K) ; \mathbb{Z}\right)$. We obtain such a presentation from the Heegaard decomposition of $\Sigma_{m}(K)$ : the generators $a_{j}^{k}$ $(0 \leq j \leq n-1,0 \leq k \leq m-1)$ corresponding to the 1 -handles dual to the $\alpha$ circles and relations corresponding to the 2-handles spanned by the $\beta$ circles. By Lemma 4.1, the relations are

$$
0=\left[\widetilde{\beta}_{i}^{k}\right]=\sum_{j=1}^{n} a_{j}^{g(i, j, k)} \quad(0 \leq i \leq n-1,0 \leq k \leq m-1) .
$$

To express $\epsilon(\mathbf{x}, \mathbf{y})$ in terms of this basis, one simply counts the number of times that a representative $\gamma_{\mathbf{x}, \mathbf{y}}$ crosses the $\alpha$ circles.

To compute the Alexander grading of a generator $\mathbf{x}$, we decompose it as $\mathbf{x}=\widetilde{\mathbf{x}}_{1} \cup \cdots \cup \widetilde{\mathbf{x}}_{m}$ using Lemma 3.1 and then use Proposition 3.5 and (2) to write:

$$
\begin{aligned}
A(\mathbf{x}) & =\frac{1}{m}\left(A\left(\mathbf{x}_{1}\right)+\cdots+A\left(\mathbf{x}_{m}\right)\right) \\
& =\frac{1}{m} \sum_{k=1}^{m}\left(a-\sum_{x \in \mathbf{x}_{k}} w(x)\right) \\
& =a-\frac{1}{m} \sum_{k=1}^{m} \sum_{x \in \tilde{\mathbf{x}}_{k}} w(\pi(x)) \\
& =a-\frac{1}{m} \sum_{x \in \mathbf{x}} w(\pi(x)) .
\end{aligned}
$$

Computing the relative Maslov grading between two generators in the same $\operatorname{spin}^{c}$ structure requires finding a domain $D$ connecting them, which is simply a matter of linear algebra, and then using the formula $M(\mathbf{x})-M(\mathbf{y})=\mu(D)-2 n_{\mathbf{w}}(D)$. The relative Maslov grading between generators in different $\operatorname{spin}^{c}$ structures can be computed similarly using the formula of Lee and Lipshitz [9]. Since all the basepoints in the Heegaard diagrams used in this paper are contained in $4 m$-gonal regions, it is not possible to compute the absolute Maslov gradings or the spectral sequence from $\widehat{\mathrm{HFK}}\left(\Sigma_{2}(K), \widetilde{K}\right)$ to $\widehat{\mathrm{HF}}\left(\Sigma_{2}(K)\right)$ combinatorially. However, when $m=2$, the groups $\widehat{\mathrm{HF}}\left(\Sigma_{2}(K)\right)$, or at least the correction terms $d\left(\Sigma_{2}(K), \mathfrak{s}\right)$, can in many instances be computed via other means (Ozsváth and Szabó [16]). In such cases, it is often possible to pin down the absolute Maslov gradings for $\widehat{\operatorname{HFK}}\left(\Sigma_{2}(K), \widetilde{K}\right)$. Specifically, the relative Maslov $\mathbb{Q}$-grading and the action of $H_{1}\left(\Sigma_{2}(K)\right)$ on $\operatorname{Spin}^{c}\left(\Sigma_{2}(K)\right)$ usually 
provide enough information to match the groups $\widehat{\operatorname{HFK}}\left(\Sigma_{2}(K), \widetilde{K}, \mathfrak{s}\right)$ up with the rational numbers $d\left(\Sigma_{2}(K), \mathfrak{s}\right)$ that are computed via some other means. If there is a $\operatorname{spin}^{c}$ structure $\mathfrak{s}$ in which $\widehat{\operatorname{HFK}}\left(\Sigma_{2}(K), \widetilde{K}, \mathfrak{s}\right)$ has rank 1 , then the absolute Maslov grading of that group equals the corresponding $d$ invariant, and the rest of the absolute gradings are completely determined.

\section{Results}

The following tables list the ranks for $\widehat{\mathrm{HFK}}\left(\Sigma_{2}(K), \widetilde{K} ; \mathbb{Z} / 2\right)$ by means of the Poincaré polynomials:

$$
p_{\mathfrak{s}}(q, t)=\sum_{i, j} \operatorname{dim}_{\mathbb{Z} / 2} \widehat{\operatorname{HFK}}_{j}\left(\Sigma_{2}(K), \tilde{K}, \mathfrak{s}, i ; \mathbb{Z} / 2\right) t^{i} q^{j} .
$$

The Maslov $\mathbb{Q}$-gradings are normalized so that in the canonical $\operatorname{spin}^{c}$ structure $\mathfrak{s}_{0}$, the nonzero elements in Alexander grading $g(K)$ have Maslov grading $g(K)$. For each knot, the first line gives $p_{\mathfrak{s}_{0}}(q, t)$, and each subsequent line gives $p_{\mathfrak{s}}(q, t)$ for a pair of conjugate $\operatorname{spin}^{c}$ structures. We identify $\operatorname{spin}^{c}$ structures with elements of $H_{1}\left(\Sigma_{2}(K) ; \mathbb{Z}\right)$, which is either a cyclic group or the sum of two cyclic groups, taking $\mathfrak{s}_{0}$ to 0 . (Of course, the choice of basis for $H_{1}\left(\Sigma_{2}(K) ; \mathbb{Z}\right)$ is not canonical.) In each $\operatorname{spin}^{c}$ structure, most of the nonzero groups lie along a single diagonal; the terms corresponding to the groups not on that diagonal are underlined.

These results were computed using a program written in $\mathrm{C}++$ and Mathematica, based on Baldwin and Gillam's program [1] for computing $\widehat{\operatorname{HFK}}\left(S^{3}, K\right)$. Most of the grid diagrams were obtained using Marc Culler's program Gridlink [2]. Using available computer resources, it was possible to compute $\widehat{\mathrm{HFK}}\left(\Sigma_{2}(K), \widetilde{K}\right)$ for all the threebridge knots with up to eleven crossings and arc index $\leq 9$, and for many knots with arc index 10. (Grigsby [6] has a much more efficient algorithm for computing $\widehat{\operatorname{HFK}}\left(\Sigma_{2}(K), \widetilde{K}\right)$ when $K$ is two-bridge, so we do not list those knots here.)

$$
\begin{array}{ccll}
K & H_{1}\left(\Sigma_{2}(K) ; \mathbb{Z}\right) & & \\
& & \sum_{i, j} \operatorname{dim}_{\mathbb{Z} / 2} \widehat{\operatorname{HFK}}_{j}\left(\Sigma_{2}(K), \widetilde{K}, \mathfrak{s}, i ; \mathbb{Z} / 2\right) t^{i} q^{j} \\
8_{5} & \mathbb{Z} / 21 & 0 & q^{-3} t^{-3}+3 q^{-2} t^{-2}+4 q^{-1} t^{-1}+5+4 q t+3 q^{2} t^{2}+q^{3} t^{3} \\
& \pm 1 & q^{5 / 21}\left(q^{-2} t^{-2}+3 q^{-1} t^{-1}+3+3 q t+q^{2} t^{2}\right) \\
& \pm 2 & q^{20 / 21} \\
& \pm 3 & q^{8 / 7} \\
& \pm 4 & q^{17 / 21}\left(q^{-1} t^{-1}+1+q t\right) \\
& \pm 5 & q^{20 / 21} \\
& \pm 6 & q^{4 / 7} \\
& \pm 7 & q^{2 / 3}\left(q^{-1} t^{-1}+3+q t\right) \\
& \pm 8 & q^{5 / 21}\left(q^{-2} t^{-2}+3 q^{-1} t^{-1}+3+3 q t+q^{2} t^{2}\right) \\
& \pm 9 & q^{2 / 7}\left(q^{-2} t^{-2}+2 q^{-1} t^{-1}+3+2 q t+q^{2} t^{2}\right) \\
& \pm 10 & q^{17 / 21}\left(q^{-1} t^{-1}+1+q t\right)
\end{array}
$$

Algebraic 83 Geometric Topology, Volume 8 (2008) 


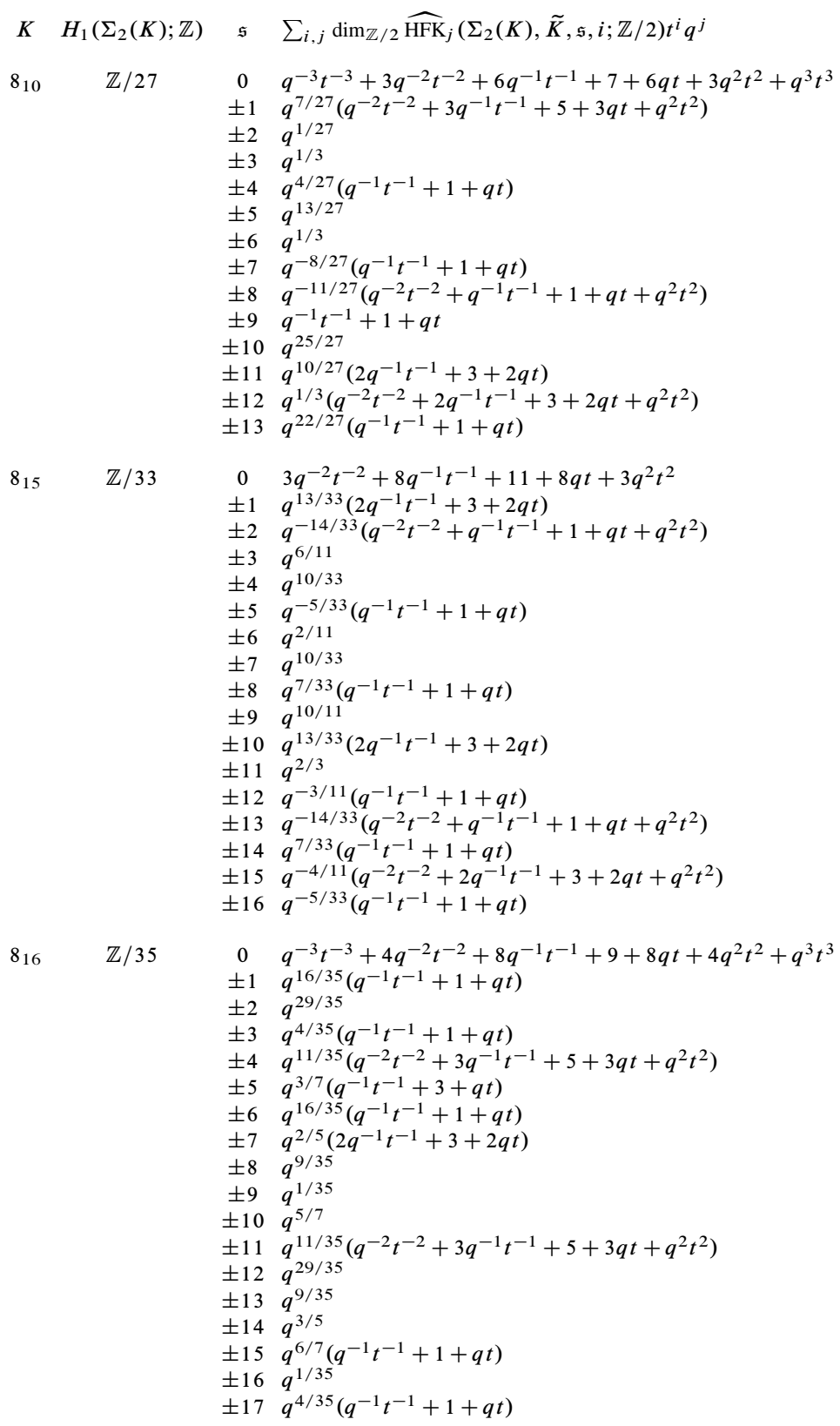




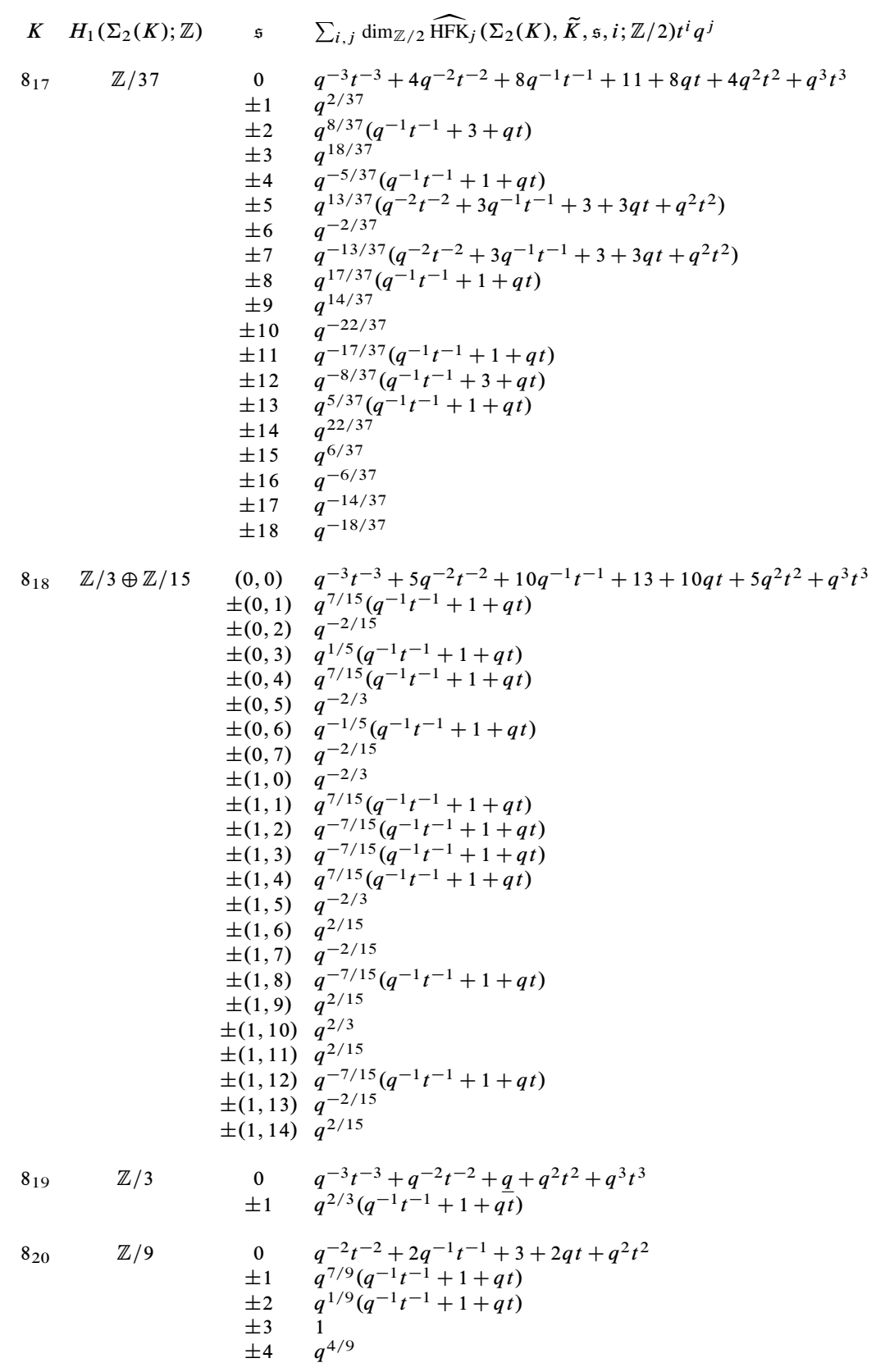




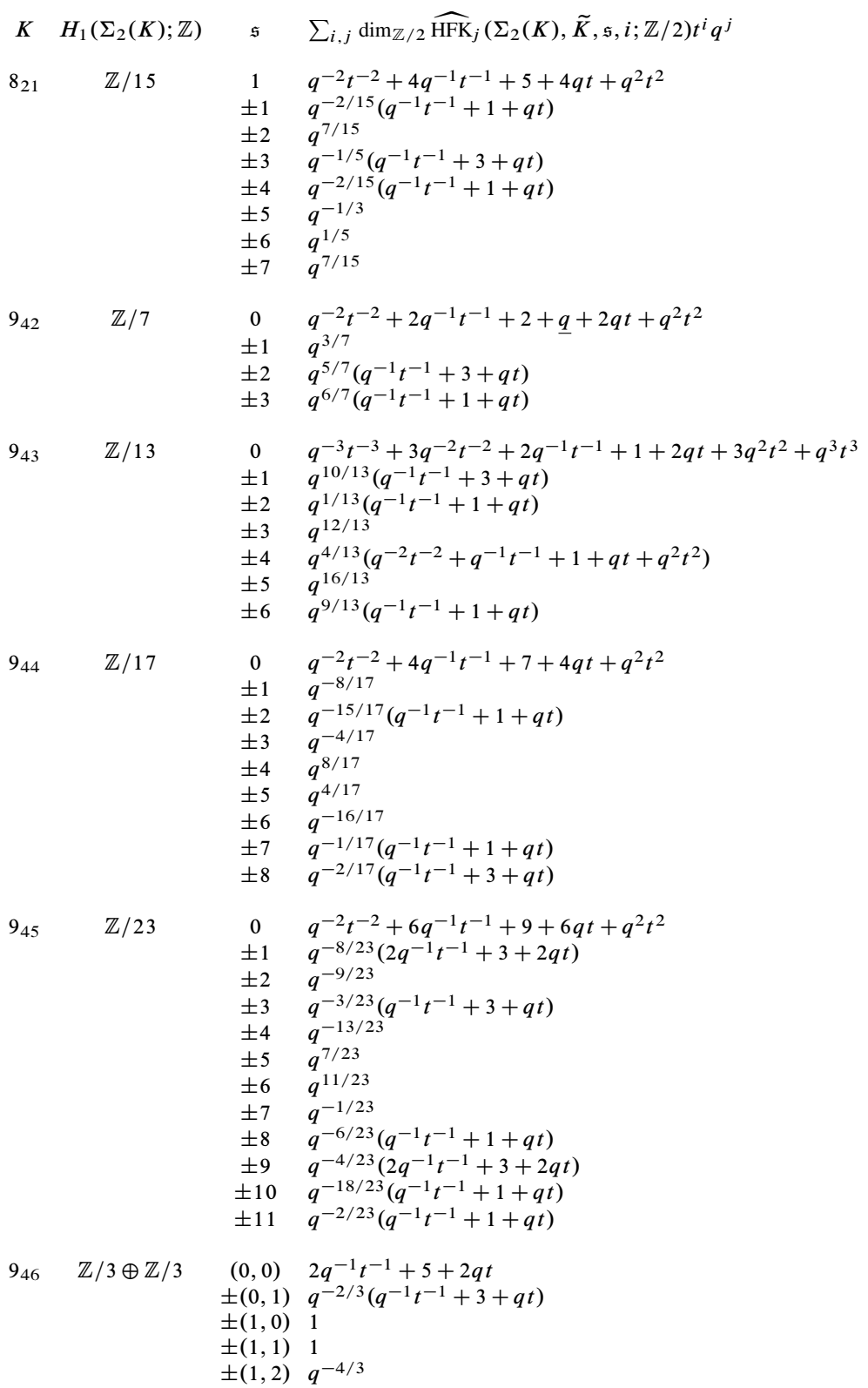




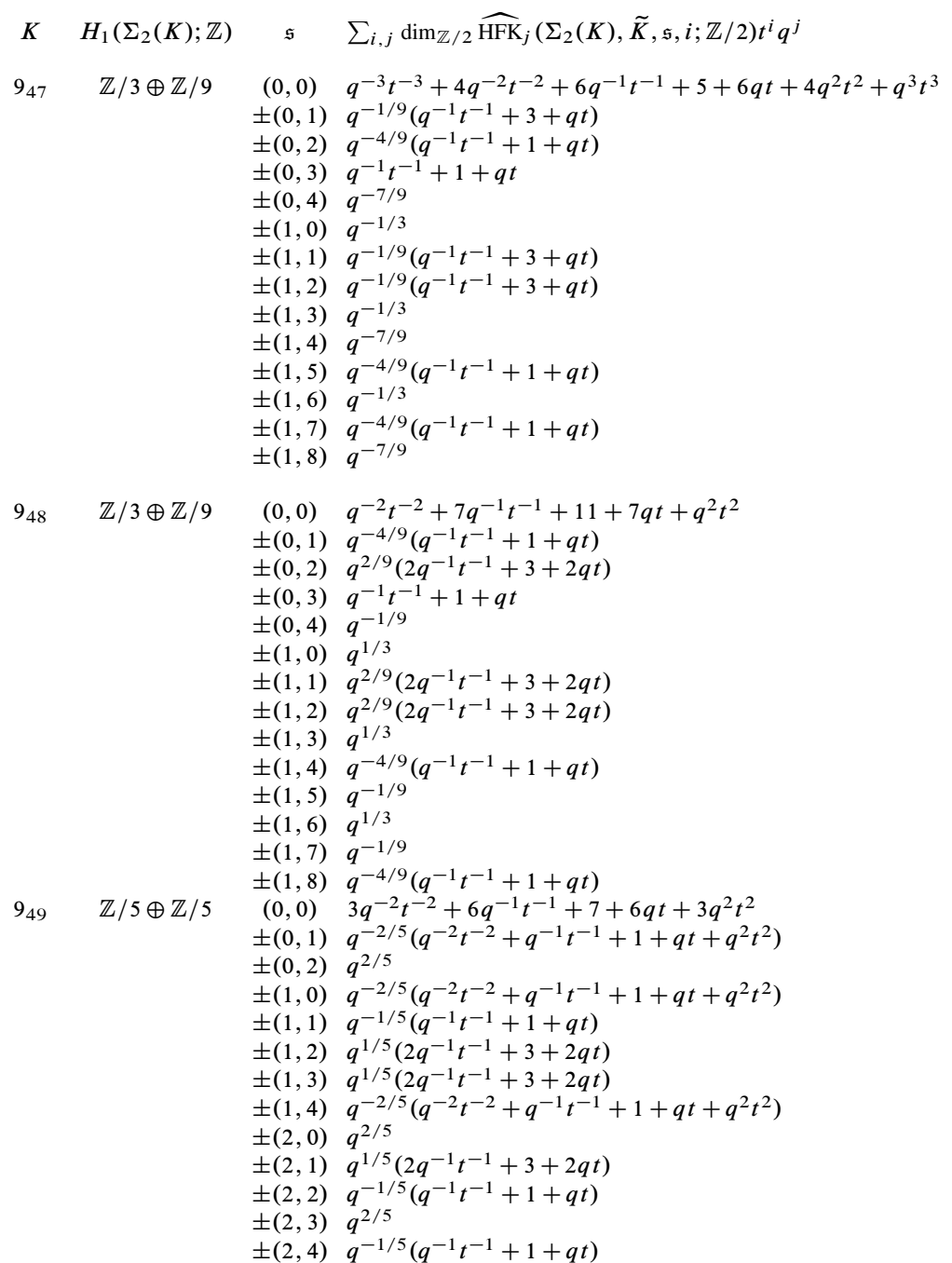

$\begin{array}{cccl}10_{124} & \{0\} & 0 & q^{-4} t^{-4}+q^{-3} t^{-3}+t^{-1}+q+q^{2} t+q^{3} t^{3}+q^{4} t^{4} \\ 10_{128} & \mathbb{Z} / 11 & 0 & 2 q^{-3} t^{-3}+3 q^{-2} t^{-2}+q^{-1} t^{-1}+q+q t+3 q^{2} t^{2}+2 q^{3} t^{3} \\ & & \pm 1 & q^{8 / 11}\left(2 q^{-1} t^{-1}+3+2 q t\right) \\ & & q^{10 / 11}\left(q^{-1} t^{-1}+1+q t\right) \\ & \pm 3 & q^{6 / 11}\left(q^{-1} t^{-1}+1+q t\right) \\ & \pm 4 & q^{-4 / 11}\left(q^{-2} t^{-2}+q^{-1} t^{-1}+q+q t+q^{2} t^{2}\right) \\ & \pm 5 & q^{2 / 11}\left(q^{-1} t^{-1}+1+q t\right)\end{array}$




\begin{tabular}{|c|c|c|c|}
\hline$K$ & $H_{1}\left(\Sigma_{2}(K) ; \mathbb{Z}\right)$ & $\mathfrak{s}$ & $\sum_{i, j} \operatorname{dim}_{\mathbb{Z} / 2} \widehat{\operatorname{HFK}}_{j}\left(\Sigma_{2}(K), \tilde{K}, \mathfrak{s}, i ; \mathbb{Z} / 2\right) t^{i} q^{j}$ \\
\hline $10_{129}$ & $\mathbb{Z} / 25$ & $\begin{array}{l}0 \\
\pm 1 \\
\pm 2 \\
\pm 3 \\
\pm 4 \\
\pm 5 \\
\pm 6 \\
\pm 7 \\
\pm 8 \\
\pm 9 \\
\pm 10 \\
\pm 11 \\
\pm 12\end{array}$ & $\begin{array}{l}2 q^{-2} t^{-2}+6 q^{-1} t^{-1}+9+6 q t+2 q^{2} t^{2} \\
q^{-8 / 25}\left(q^{-2} t^{-2}+2 q^{-1} t^{-1}+3+2 q t+q^{2} t^{2}\right) \\
q^{-7 / 25}\left(q^{-1} t^{-1}+1+q t\right) \\
q^{3 / 25}\left(2 q^{-1} t^{-1}+3+2 q t\right) \\
q^{-3 / 25}\left(q^{-1} t^{-1}+1+q t\right) \\
1 \\
q^{12 / 25} \\
q^{8 / 25} \\
q^{-12 / 25} \\
q^{2 / 25}\left(q^{-1} t^{-1}+3+q t\right) \\
1 \\
q^{7 / 25}\left(q^{-1} t^{-1}+1+q t\right) \\
q^{23 / 25}\left(q^{-1} t^{-1}+1+q t\right)\end{array}$ \\
\hline $10_{130}$ & $\mathbb{Z} / 17$ & $\begin{array}{l}0 \\
\pm 1 \\
\pm 2 \\
\pm 3 \\
\pm 4 \\
\pm 5 \\
\pm 6 \\
\pm 7 \\
\pm 8\end{array}$ & $\begin{array}{l}2 q^{-2} t^{-2}+4 q^{-1} t^{-1}+5+4 q t+2 q^{2} t^{2} \\
q^{4 / 17}\left(q^{-2} t^{-2}+2 q^{-1} t^{-1}+3+2 q t+q^{2} t^{2}\right) \\
q^{16 / 17} \\
q^{19 / 17}\left(q^{-1} t^{-1}+1+q t\right) \\
q^{13 / 17}\left(2 q^{-1} t^{-1}+3+2 q t\right) \\
q^{15 / 17}\left(q^{-1} t^{-1}+1+q t\right) \\
q^{8 / 17} \\
q^{9 / 17}\left(q^{-1} t^{-1}+1+q t\right) \\
q^{1 / 17}\left(q^{-1} t^{-1}+1+q t\right)\end{array}$ \\
\hline $10_{131}$ & $\mathbb{Z} / 31$ & $\begin{array}{l}0 \\
\pm 1 \\
\pm 2 \\
\pm 3 \\
\pm 4 \\
\pm 5 \\
\pm 6 \\
\pm 7 \\
\pm 8 \\
\pm 9 \\
\pm 10 \\
\pm 11 \\
\pm 12 \\
\pm 13 \\
\pm 14 \\
\pm 15\end{array}$ & $\begin{array}{l}2 q^{-2} t^{-2}+8 q^{-1} t^{-1}+11+8 q t+2 q^{2} t^{2} \\
q^{-18 / 31}\left(q^{-1} t^{-1}+1+q t\right) \\
q^{-10 / 31}\left(q^{-1} t^{-1}+1+q t\right) \\
q^{-7 / 31}\left(q^{-1} t^{-1}+3+q t\right) \\
q^{-9 / 31} \\
q^{15 / 31} \\
q^{3 / 31} \\
q^{-14 / 31}\left(q^{-1} t^{-1}+1+q t\right) \\
q^{-5 / 31}\left(2 q^{-1} t^{-1}+5+2 q t\right) \\
q^{-1 / 31} \\
q^{-2 / 31}\left(q^{-1} t^{-1}+1+q t\right) \\
q^{-8 / 31}\left(q^{-2} t^{-2}+4 q^{-1} t^{-1}+5+4 q t+q^{2} t^{2}\right) \\
q^{-19 / 31}\left(q^{-1} t^{-1}+3+q t\right) \\
q^{-4 / 31}\left(2 q^{-1} t^{-1}+3+2 q t\right) \\
q^{-25 / 31} \\
q^{11 / 31}\end{array}$ \\
\hline $10_{132}$ & $\mathbb{Z} / 5$ & $\begin{array}{l}0 \\
\pm 1 \\
\pm 2\end{array}$ & $\begin{array}{l}q^{-2} t^{-2}+\left(2 q^{-1}+1\right) t^{-1}+(2+\underline{q})+\left(2 q+\underline{q^{2}}\right) t+q^{2} t^{2} \\
q^{2 / 5} \\
q^{3 / 5}\left(q^{-1} t^{-1}+1+q t\right)\end{array}$ \\
\hline $10_{133}$ & $\mathbb{Z} / 19$ & $\begin{array}{l}0 \\
\pm 1 \\
\pm 2 \\
\pm 3 \\
\pm 4 \\
\pm 5 \\
\pm 6 \\
\pm 7 \\
\pm 8 \\
\pm 9\end{array}$ & $\begin{array}{l}q^{-2} t^{-2}+5 q^{-1} t^{-1}+7+5 q t+q^{2} t^{2} \\
q^{-3 / 19} \\
q^{-12 / 19}\left(q^{-1} t^{-1}+1+q t\right) \\
q^{-8 / 19}\left(q^{-1} t^{-1}+1+q t\right) \\
q^{9 / 19} \\
q^{1 / 19} \\
q^{-13 / 19}\left(q^{-1} t^{-1}+3+q t\right) \\
q^{5 / 19} \\
q^{-2 / 19}\left(2 q^{-1} t^{-1}+3+2 q t\right) \\
q^{-15 / 19}\end{array}$ \\
\hline
\end{tabular}




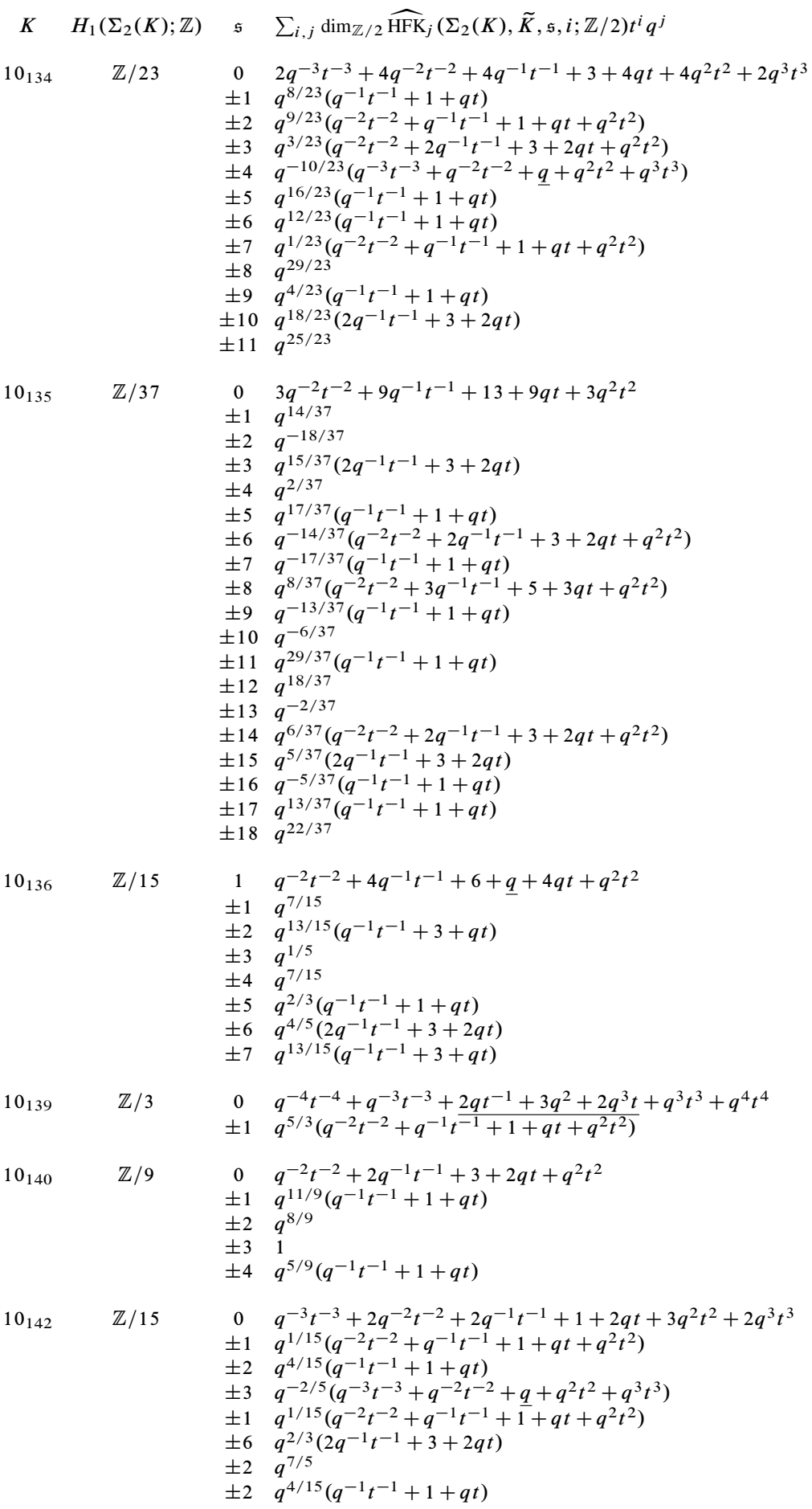


$K \quad H_{1}\left(\Sigma_{2}(K) ; \mathbb{Z}\right) \quad \mathfrak{s} \quad \sum_{i, j} \operatorname{dim}_{\mathbb{Z} / 2} \widehat{\mathrm{HFK}}_{j}\left(\Sigma_{2}(K), \tilde{K}, \mathfrak{s}, i ; \mathbb{Z} / 2\right) t^{i} q^{j}$

$10_{145} \quad \mathbb{Z} / 3 \quad 0 \quad q^{-2} t^{-2}+\left(q^{-1}+\underline{2 q}\right) t^{-1}+\underline{q+4 q^{2}}+\left(q+\underline{2 q^{3}}\right) t+q^{2} t^{2}$

$\pm 1 \quad q^{4 / 3}\left(2 q^{-1} t^{-1}+3+2 q t\right)$

$10_{147} \quad \mathbb{Z} / 27 \quad 0 \quad 2 q^{-2} t^{-2}+7 q^{-1} t^{-1}+9+7 q t+2 q^{2} t^{2}$

$\pm 1 \quad q^{7 / 27}\left(q^{-1} t^{-1}+3+q t\right)$

$\pm 2 \quad q^{1 / 27}$

$\pm 3 \quad q^{1 / 3}\left(2 q^{-1} t^{-1}+5+2 q t\right)$

$\pm 4 \quad q^{4 / 27}\left(q^{-2} t^{-2}+3 q^{-1} t^{-1}+3+3 q t+q^{2} t^{2}\right)$

$\pm 5 \quad q^{13 / 2}$

$\pm 6 \quad q^{1 / 3}$

$\pm 7 \quad q^{19 / 27}\left(q^{-1} t^{-1}+3+q t\right)$

$\pm 8 \quad q^{16 / 27}\left(q^{-1} t^{-1}+1+q t\right)$

$\pm 9 \quad q^{-1} t^{-1}+1+q t$

$\pm 10 \quad q^{25 / 27}$

$\pm 11 \quad q^{37 / 27}$

$\pm 12 q^{1 / 3}$

$\pm 13 \quad q^{22 / 27}\left(2 q^{-1} t^{-1}+3+2 q t\right)$

$10_{158} \quad \mathbb{Z} / 45$

$\begin{array}{cl}0 & q^{-3} t^{-3}+4 q^{-2} t^{-2}+10 q^{-1} t^{-1}+15+10 q t+4 q^{2} t^{2}+q^{3} t^{3} \\ \pm 1 & q^{8 / 45}\left(q^{-1} t^{-1}+3+q t\right)\end{array}$

$\pm 2 \quad q^{-13 / 45}\left(q^{-2} t^{-2}+3 q^{-1} t^{-1}+3+3 q t+q^{2} t^{2}\right)$

$\pm 3 \quad q^{-2 / 5}$

$\pm 4 \quad q^{38 / 45}$

$\pm 5 \quad q^{4 / 9}\left(q^{-1} t^{-1}+3+q t\right)$

$\pm 6 \quad q^{2 / 5}$

$\pm 7 \quad q^{-13 / 45}\left(q^{-2} t^{-2}+3 q^{-1} t^{-1}+3+3 q t+q^{2} t^{2}\right)$

$\pm 8 \quad q^{17 / 45}\left(q^{-2} t^{-2}+3 q^{-1} t^{-1}+3+3 q t+q^{2} t^{2}\right)$

$\pm 9 \quad q^{2 / 5}\left(2 q^{-1} t^{-1}+5+2 q t\right)$

$\pm 10 q^{-2 / 9}\left(2 q^{-1} t^{-1}+5+2 q t\right)$

$\pm 11 q^{-22 / 45}$

$\pm 12 q^{-2 / 5}$

$\pm 13 \quad q^{2 / 45}$

$\pm 14 q^{38 / 45}$

$\pm 15 q^{-1} t^{-1}+3+q t$

$\pm 16 \quad q^{-22 / 45}$

$\pm 17 \quad q^{17 / 45}\left(q^{-2} t^{-2}+3 q^{-1} t^{-1}+3+3 q t+q^{2} t^{2}\right)$

$\pm 18 \quad q^{-2 / 5}$

$\pm 19 q^{8 / 45}\left(q^{-1} t^{-1}+3+q t\right)$

$\pm 20 \quad q^{1 / 9}\left(q^{-1} t^{-1}+1+q t\right)$

$\pm 21 q^{2 / 5}$

$\pm 22 q^{2 / 45}$

$10_{160}$

$\mathbb{Z} / 21$

$0 \quad q^{-3} t^{-3}+4 q^{-2} t^{-2}+4 q^{-1} t^{-1}+3+4 q t+4 q^{2} t^{2}+q^{3} t^{3}$

$\pm 1 \quad q^{1 / 21}\left(q^{-1} t^{-1}+1+q t\right)$

$\pm 2 \quad q^{4 / 21}\left(q^{-2} t^{-2}+q^{-1} t^{-1}+1+q t+q^{2} t^{2}\right)$

$\pm 3 \quad q^{3 / 7}\left(q^{-1} t^{-1}+1+q t\right)$

$\pm 4 \quad q^{16 / 21}$

$\pm 5 \quad q^{4 / 21}\left(q^{-2} t^{-2}+q^{-1} t^{-1}+1+q t+q^{2} t^{2}\right)$

$\pm 6 \quad q^{5 / 7}\left(2 q^{-1} t^{-1}+3+2 q t\right)$

$\pm 7 \quad q^{4 / 3}$

$\pm 8 \quad q^{1 / 21}\left(q^{-1} t^{-1}+1+q t\right)$

$\pm 9 \quad q^{6 / 7}\left(q^{-1} t^{-1}+3+q t\right)$

$\pm 10 q^{16 / 21}$

$10_{161} \quad \mathbb{Z} / 5$

$\mathbb{Z} / 5$

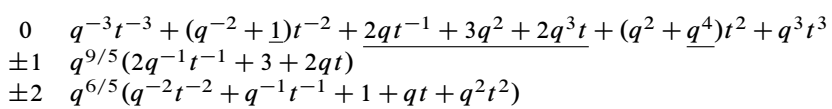

Algebraic $\&$ Geometric Topology, Volume 8 (2008) 


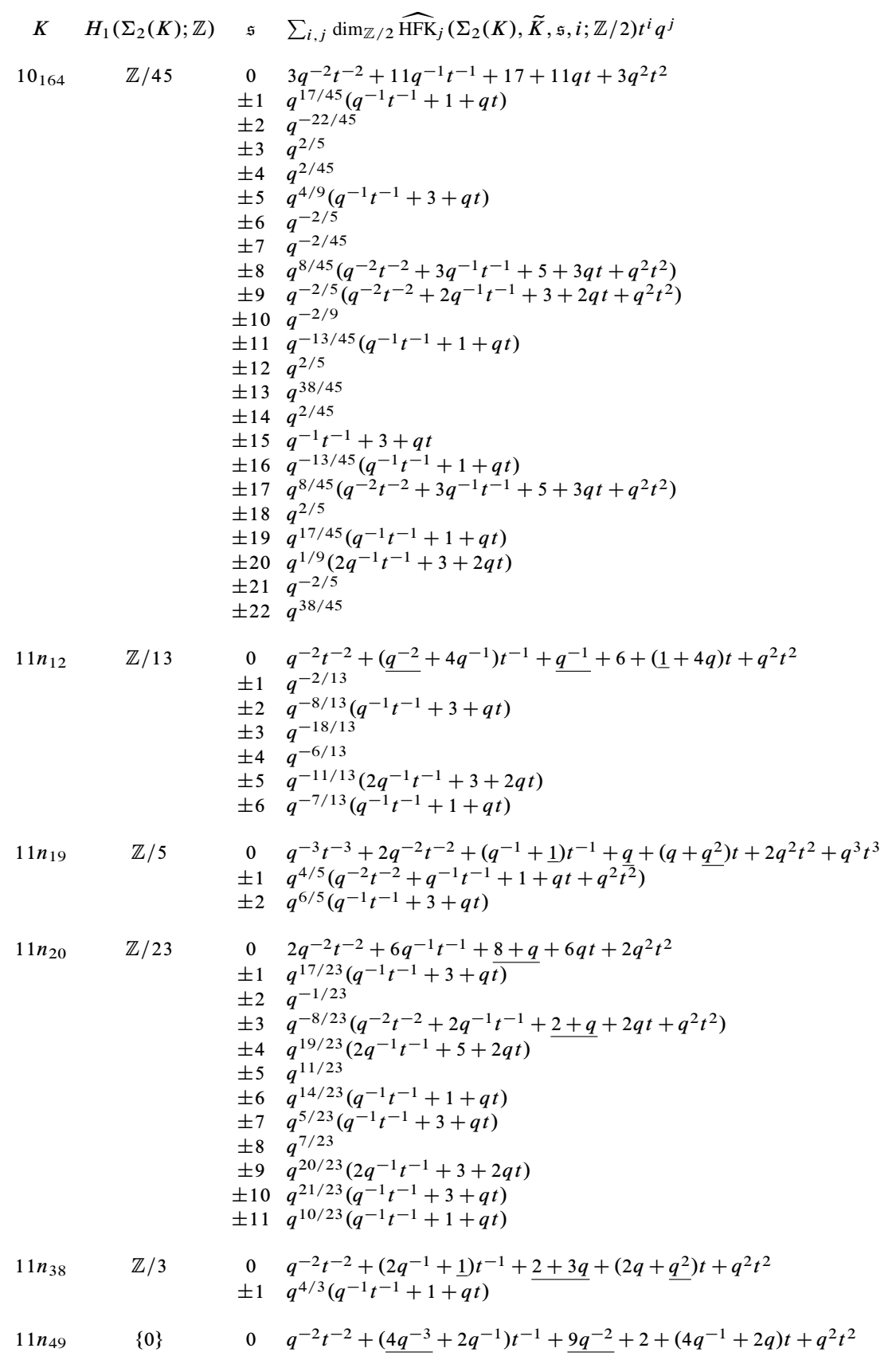




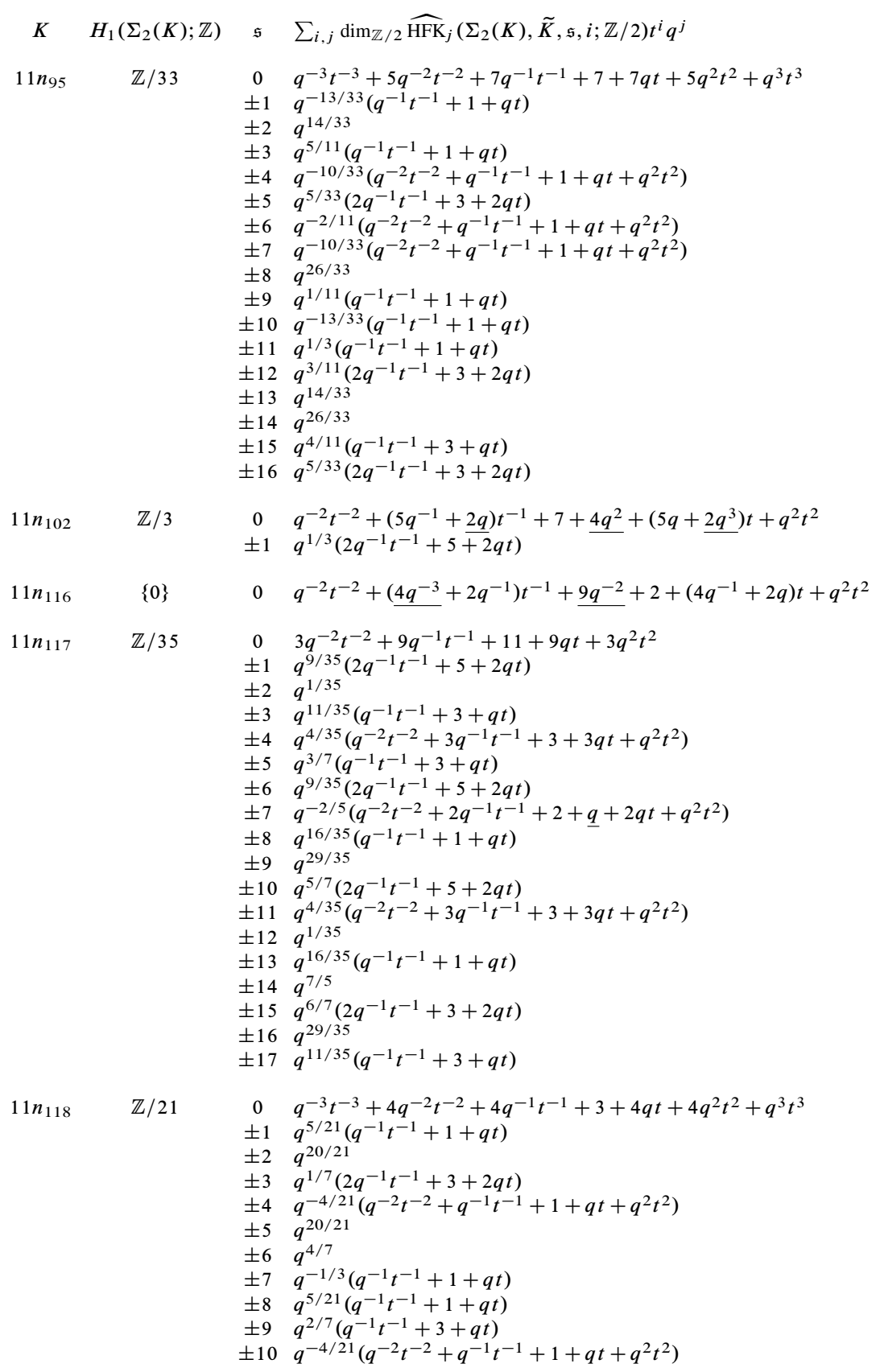




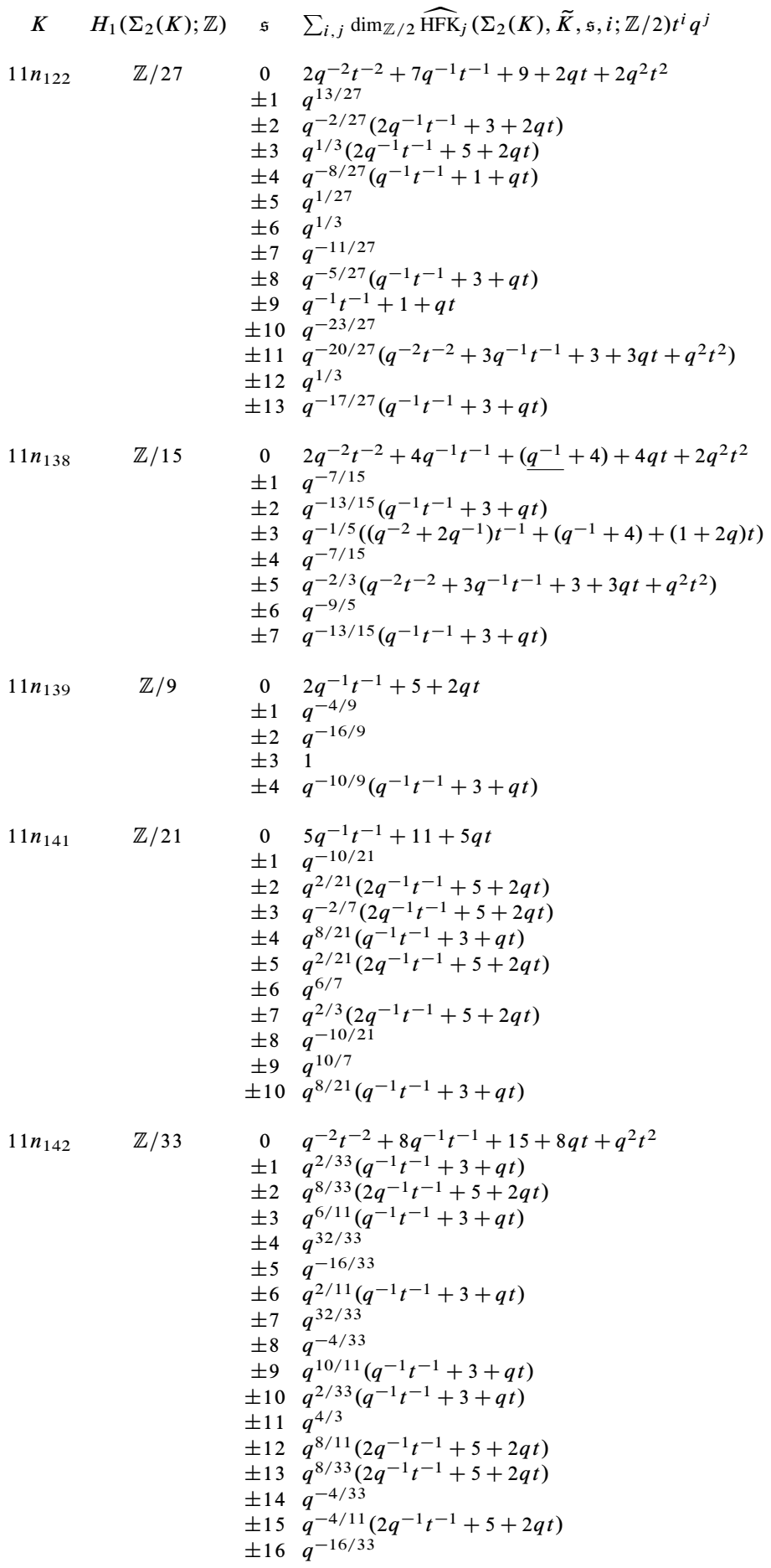




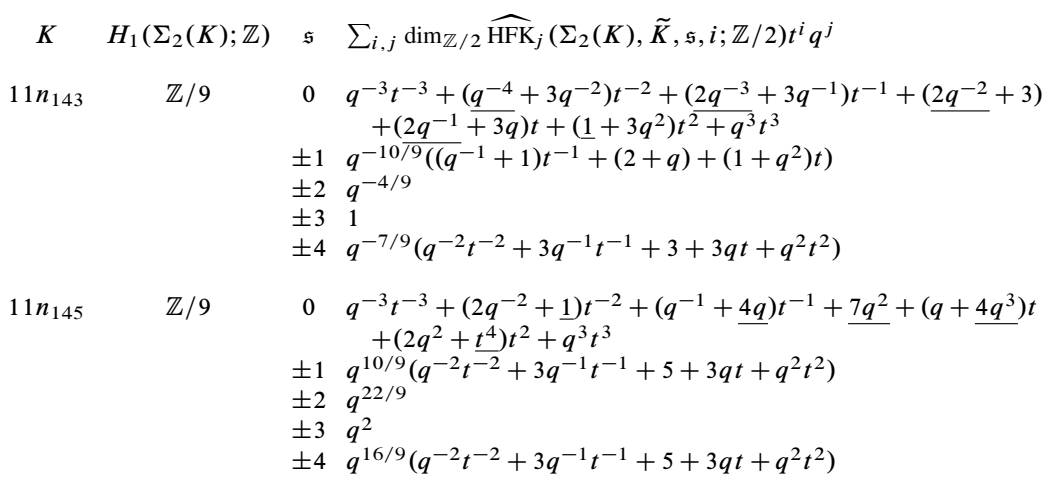

\section{Observations}

Grigsby [7] showed that when $K \subset S^{3}$ is a two-bridge knot, the Heegaard Floer knot homology of $\widetilde{K} \subset \Sigma_{2}(K)$ in the canonical $\operatorname{spin}^{c}$ structure is isomorphic as a bigraded $\mathbb{Z} / 2$-vector space to that of $K \subset S^{3}$ : ie, $\widehat{\operatorname{HFK}}\left(\Sigma_{2}(K), \widetilde{K}, \mathfrak{s}_{0}\right) \cong \widehat{\operatorname{HFK}}\left(S^{3}, K\right)$, up to an overall shift in the Maslov grading. Our results suggest that the same is true for a wider class of knots. Specifically, define the $\delta$-grading on $\widehat{\operatorname{HFK}}(Y, K, \mathfrak{s})$ as the difference between the Alexander and Maslov gradings. We say that $\widehat{\operatorname{HFK}}(Y, K, \mathfrak{s})$ is thin if it is supported in a single $\delta$-grading. We make the following conjecture.

Conjecture 6.1 Let $K \subset S^{3}$ be a knot for which $\widehat{\operatorname{HFK}}\left(S^{3}, K\right)$ is thin. Then

$$
\widehat{\operatorname{HFK}}\left(\Sigma_{2}(K), \widetilde{K}, \mathfrak{s}_{0}\right) \cong \widehat{\operatorname{HFK}}\left(S^{3}, K\right)
$$

as bigraded groups, up to a possible shift in the absolute Maslov grading.

It is well-known (Ozsváth-Szabó [14] or Rasmussen [17]) that $\widehat{\operatorname{HFK}}\left(S^{3}, K\right)$ is thin whenever $K$ is alternating (and hence for all two-bridge knots). More generally, let $\mathcal{Q}$ be the smallest set of link types such that:

- The unknot is in $\mathcal{Q}$.

- Suppose $L$ admits a projection such that the two resolutions at some crossing, $L_{0}$ and $L_{1}$, are both in $\mathcal{Q}$ and satisfy $\operatorname{det}\left(L_{0}\right)+\operatorname{det}\left(L_{1}\right)=\operatorname{det}(L)$. Then $L$ is in $\mathcal{Q}$.

The links in $\mathcal{Q}$ are called quasi-alternating; for instance, any alternating link is quasialternating. Manolescu and Ozsváth [11] that whenever $L$ is quasi-alternating, both $\widehat{\operatorname{HFK}}\left(S^{3}, L\right)$ and the Khovanov homology of $L$ are thin. Conjecture 6.1 would then imply that $\widehat{\operatorname{HFK}}\left(\Sigma_{2}(K), \widetilde{K}, \mathfrak{s}_{0}\right)$ is thin whenever $K$ is quasi-alternating. 

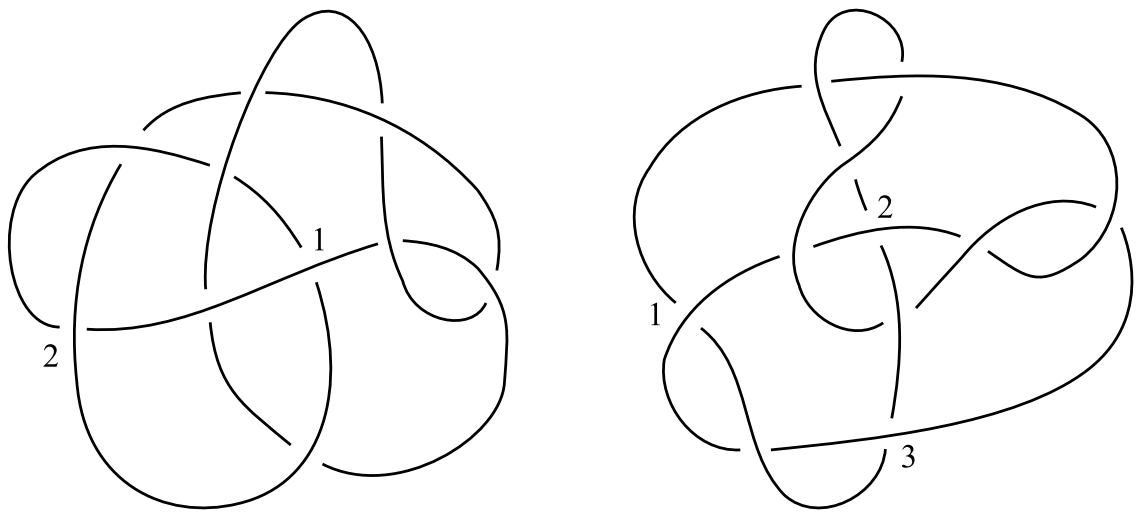

Figure 2: To see that the knots $10_{134}$ (left) and $11 n_{117}$ (right) are quasialternating, resolve the marked crossings in the order indicated.

One may also ask under what conditions $\widehat{\mathrm{HFK}}\left(\Sigma_{2}(K), \widetilde{K}, \mathfrak{s}\right)$ is thin for $\operatorname{spin}^{c}$ structures $\mathfrak{s} \neq \mathfrak{s}_{0}$. The knots $10_{134}$ and $11 n_{117}$ both satisfy the hypothesis and conclusion of Conjecture 6.1. Indeed, they are both quasi-alternating, as illustrated in Figure 2. However, each one admits a $\operatorname{spin}^{c}$ structure $\mathfrak{s}$ on $\Sigma_{2}(K)$ in which $\widehat{\operatorname{HFK}}\left(S^{2}(K), \widetilde{K}, \mathfrak{s}\right)$ is not thin. There are no known examples of alternating knots for which this phenomenon occurs, though.

On the other hand, when $\widehat{\operatorname{HFK}}\left(S^{3}, K\right)$ is not thin, the isomorphism between $\widehat{\operatorname{HFK}}\left(S^{3}, K\right)$ and $\widehat{\operatorname{HFK}}\left(\Sigma_{2}(K), \widetilde{K}, \mathfrak{s}_{0}\right)$ generally fails. A few patterns are worth mentioning. Note that for the knots considered here, in each Alexander grading $i$, the total rank of $\widehat{\operatorname{HFK}}\left(\Sigma_{2}(K), \widetilde{K}, \mathfrak{s}_{0}, i\right)$ is at least that of $\widehat{\operatorname{HFK}}\left(S^{3}, K, i\right)$, and the two ranks are congruent modulo 2. Some examples in which the ranks fail to be equal are $11 n_{49}, 11 n_{102}$, and $11 n_{116}$. Even when the total ranks of $\widehat{\operatorname{HFK}}\left(\Sigma_{2}(K), \widetilde{K}, \mathfrak{s}_{0}, i\right)$ and $\widehat{\operatorname{HFK}}\left(S^{3}, K, i\right)$ are the same for all $i$, the relative Maslov gradings can differ. A common pattern is that the Maslov gradings of all the groups in one $\delta$-grading of $\widehat{\operatorname{HFK}}\left(S^{3}, K\right)$ are shifted by a constant amount in $\widehat{\operatorname{HFK}}\left(\Sigma_{2}(K), \widetilde{K}, \mathfrak{s}_{0}\right)$, such as with the knots $9_{42}$ and $10_{161}$, where the groups are shifted by 2 and 3, respectively. However, there are also examples where the relative Maslov gradings in different Alexander gradings change in different ways. For example, for $10_{145}$, the total ranks of $\widehat{\operatorname{HFK}}\left(S^{3}, K, i\right)$ and $\widehat{\mathrm{HFK}}\left(\Sigma_{2}(K), \widetilde{K}, \mathfrak{s}_{0}, i\right)$ are the same for each $i$, but $\widehat{\mathrm{HFK}}\left(S^{3}, K\right)$ is supported in two $\delta$-gradings while $\widehat{\operatorname{HFK}}\left(\Sigma_{2}(K), K, \mathfrak{s}_{0}\right)$ is supported in three.

Finally, note that the pretzel knots $8_{20}=P(2,3,-3)$ and $10_{140}=P(4,3,-3)$ have identical knot Floer homology but can be distinguished by $\widehat{\operatorname{HFK}}\left(\Sigma_{2}(K), \widetilde{K}\right)$. The relative Maslov gradings between $\operatorname{spin}^{c}$ structures are necessary in this case. For another such example, see Grigsby [7]. 


\section{References}

[1] J A Baldwin, W D Gillam, Computations of Heegaard Floer knot homology arXiv: math/0610167

[2] M Culler, Gridlink: a tool for knot theorists www. math.uic.edu/ culler/gridlink/

[3] R Diestel, Graph theory, third edition, Graduate Texts in Mathematics 173, Springer, Berlin (2005) MR2159259

[4] R H Fox, A quick trip through knot theory, from: "Topology of 3-manifolds and related topics (Proc. The Univ. of Georgia Institute, 1961)”, Prentice-Hall, Englewood Cliffs, N.J. (1962) 120-167 MR0140099

[5] C M Gordon, Some aspects of classical knot theory, from: "Knot theory (Proc. Sem., Plans-sur-Bex, 1977)", Lecture Notes in Math. 685, Springer, Berlin (1978) 1-60 MR521730

[6] JE Grigsby, Combinatorial description of knot Floer homology of cyclic branched covers arXiv:math/0610238

[7] J E Grigsby, Knot Floer homology in cyclic branched covers, Algebr. Geom. Topol. 6 (2006) 1355-1398 MR2253451

[8] J Grigsby, D Ruberman, S Strle, Knot concordance and Heegaard Floer homology invariants in branched covers arXiv:math/0701460

[9] D A Lee, R Lipshitz, Covering spaces and $\mathbb{Q}$-gradings on Heegaard Floer homology arXiv: math/0608001

[10] R Lipshitz, A cylindrical reformulation of Heegaard Floer homology, Geom. Topol. 10 (2006) 955-1097 MR2240908

[11] C Manolescu, P Ozsváth, On the Khovanov and knot Floer homologies of quasialternating links arXiv:math/0708.3249v1

[12] C Manolescu, P Ozsváth, S Sarkar, A combinatorial description of knot Floer homology arXiv:math/0607691

[13] C Manolescu, P Ozsváth, Z Szabó, D Thurston, On combinatorial link Floer homology arXiv:math/0610559

[14] P Ozsváth, Z Szabó, Heegaard Floer homology and alternating knots, Geom. Topol. 7 (2003) 225-254 MR1988285

[15] P Ozsváth, Z Szabó, Holomorphic disks and knot invariants, Adv. Math. 186 (2004) 58-116 MR2065507

[16] P Ozsváth, Z Szabó, Knots with unknotting number one and Heegaard Floer homology, Topology 44 (2005) 705-745 MR2136532

[17] J A Rasmussen, Floer homology of surgeries on two-bridge knots, Algebr. Geom. Topol. 2 (2002) 757-789 MR1928176

Algebraic 8 Geometric Topology, Volume 8 (2008) 
[18] J A Rasmussen, Floer homology and knot complements, $\mathrm{PhD}$ thesis, Harvard University (2003) arXiv:math/0306378

[19] D Rolfsen, Knots and links, Mathematics Lecture Series 7, Publish or Perish, Houston, TX (1990) MR1277811

[20] S Sarkar, J Wang, A combinatorial description of some Heegaard Floer homologies arXiv:math/0607777

Department of Mathematics, Columbia University

2990 Broadway, New York, NY 10027, USA

alevine@math.columbia.edu

Received: 9 December 2007 Revised: 4 March 2008 